\title{
Global Supply Chain Network Dynamics with Multicriteria Decision-Making Under Risk and Uncertainty
}

\author{
Anna Nagurney and Dmytro Matsypura* \\ Department of Finance and Operations Management \\ Isenberg School of Management \\ University of Massachusetts \\ Amherst, Massachusetts 01003
}

submitted June 2004; revised December 2004; revised May 2005

Appears in Transportation Research E 41 (2005), pp. 585-612.

\begin{abstract}
In this paper, we consider the dynamics of a global supply chain network economy in the presence of risk and uncertainty in which distinct speeds of adjustment are included. We assume three tiers of decision-makers: manufacturers, distributors, and retailers, who acquire the product in order to satisfy the demand at the demand markets. The manufacturers, distributors, and retailers may be based in the same or in different countries and may transact in different currencies. We allow for electronic transactions in the form of electronic commerce between the manufacturers and the retailers as well as between the distributors and the retailers since the retailers may be physical or virtual. In addition, supply-side risk and demand-side risk are handled in our formulation with the former being expressed as a multicriteria decision-making problem for each manufacturer and distributor (with distinct weights associated with the criteria) and the latter being handled with the use of uncertain demands. The proposed framework allows for the modeling and theoretical analysis of such global supply chain networks, which involve competition within a tier of decision-makers but cooperation between tiers. Numerical examples are provided for illustrative purposes.
\end{abstract}

Key words: global supply chains, multicriteria decision-making, variational inequalities, projected dynamical systems

* This research was supported in part by NSF Grant No.: IIS-0002647 and by an AT\&T Industrial Ecology Fellowship. This support is gratefully acknowledged. The authors are indebted to the reviewers and to the Guest Editor, Professor Bookbinder, for numerous comments and suggestions on earlier versions of this paper. The authors acknowledge Professors June Dong and Jose Cruz for helpful discussions. 


\section{Introduction}

Fewer than ten years ago, the purchase of a product, typically, involved getting dressed, using some mode of transport to reach the shopping destination, selecting the product physically, and then paying for the purchase. Today, many consumers, be they at home, at work, or at some other location, can go online 24 hours a day, 7 days a week, and select and purchase a great assortment of products over the Internet while doing their "shopping." Indeed, it is now possible, in many instances, to buy books, videos, and CDs online as well as to order food from a supermarket or restaurant in an electronic manner, and have the items delivered afterwards to the desired destination.

The revolution in electronic commerce has affected not only consumers and their decisionmaking but has also influenced the producers/manufacturers, the distributors, as well as the retailers (be they physical or virtual), and, in effect, the entire product supply chain. According to Salkever (2003), Intel, one of the world's largest computer chip manufacturers, last year generated $85 \%$ of its orders - some $\$ 22.8$ billion worth - online. Moreover, $26 \%$ of the sales occurred after the company's physical offices had closed for the day. Furthermore, Intel estimates that it saved $\$ 500$ million in the year 2002 due to greater control over the supplies that it requires for chip manufacture.

Indeed, both business-to-consumer (B2C) commerce and business-to-business (B2B) commerce via the Internet are thriving. For example, in Europe alone, the amount of goods and services purchased online by companies in 2002 surpassed $\$ 200$ billion (cf. Reinhardt and Majidi (2003)), resulting in a $100 \%$ growth in the number of transactions, with B2C commerce in Europe growing at an annual rate of $75 \%$.

The advent of electronic commerce is enabling the world to move closer to the realization of a single, borderless market and is driving the increasing globalization of not only businesses but also supply chains. At the same time, however, increasing globalization exposes supply chains to new risks and uncertainties. For example, recently, the threat of illness in the form of SARS (see Engardio et al. (2003)) disrupted supply chains as have terrorist threats and wars (see Sheffi (2001)). Frameworks for risk management in a global supply chain context with a focus on centralized decision-making and optimization have been proposed by Huchzermeier and Cohen (1996), Cohen and Mallik (1997), and Cohen and Huchzermeier (1998) (see also the references therein). Indeed, although the importance of global issues in supply chain management and analysis has been emphasized in several papers (cf. Kogut and Kulatilaka (1994), Cohen and Mallik (1997), Nagurney, Cruz, and Matsypura (2003)), the topic of supply chain risk modeling is fairly new and requires novel methodological 
approaches that are able to capture the operational as well as the financial aspects of such decision-making.

In addition, it is imperative to be able to address dynamic issues since not only does the speed of the Internet allow for transactions to be conducted across the globe but such transactions can occur very rapidly. Moreover, it is important to recognize and capture distinct speeds associated with the decision-makers' transactions. In 1939, Hicks in his classic work took the first steps towards the integration of comparative statics and dynamic economics. Samuelson (1941), subsequently, in a pioneering article presented the first complete synthesis of dynamics and comparative statics and demonstrated the importance of dynamic economics in the analysis of equilibrium. In these publications, as well as in numerous studies that followed (see, e.g., Metzler (1945), Arrow and Hurwicz (1958), Quandt (1967), Hahn (1970), and Okuguchi (1976)), there was one natural and, yet very important, assumption made: a change in price is proportional to the difference between the demand and the supply of the product. Fisher (1961) offered a similar adjustment assumption for the individual outputs and suggested that it might have interesting consequences for the discussion of stability.

In this paper, we build upon the ideas of classical economics in terms of dynamics by proposing a dynamic global supply chain network model which includes explicit speeds of adjustment, which are incorporated into a projected dynamical systems formalism (cf. Dupuis and Nagurney (1993), Zhang and Nagurney (1995), and Nagurney and Zhang (1996)) for the first time. The dynamic global supply chain network model also captures decision-making under risk and uncertainty associated with both the supply-side as well as the demand-side. The model is accompanied by entirely new theoretical results.

In particular, in this paper, we focus on the development of a dynamic global supply chain supernetwork model with supply-side and demand-side risk that considers the interactions among three distinct tiers of decision-makers, notably, the manufacturers, the distributors, as well as the retailers, who must respond to the consumers as represented by the associated demand functions for the product. The speeds of adjustment may be distinct for each transaction that takes place. The general framework that we utilize is that of supernetworks, as introduced by Nagurney and Dong (2002) to capture the trade-offs associated with decision-making on transportation versus telecommunication networks in the Information Age.

This paper is organized as follows. In Section 2, we develop the global supply chain network model in which distinct speeds of adjustment are allowed and derive its projected dynamical system formulation. The projected dynamical system describes how the product 
transactions evolve through time between the tiers of the "supernetwork" as well as how the prices associated with the retailers and the distributors evolve. We also establish that the set of stationary points of the projected dynamical system coincides with the set of equilibrium points (formulated as a variational inequality problem). In the same section, we provide some theoretical properties of the projected dynamical system and prove that the set of equilibria is independent of the speeds of adjustment. In Section 3, we propose an algorithm, which provides a time-discretization of the continuous time trajectories of the product transactions and prices. In Section 4, we apply the algorithm to several numerical global supply chain network examples. The numerical results support the theoretical results established in this paper. We conclude the paper with a summary and discussion in Section 5. 


\section{The Dynamic Global Supply Chain Network Model with Risk and Uncertainty}

In this Section, we develop the dynamic global supply chain network model in which physical and electronic transactions are allowed and in which both supply-side and demandside risks are included. In particular, we consider (cf. Figure 1) a three tiered supply chain network consisting of: manufacturers, distributors, and retailers, and these are associated, respectively, with the top tier, the middle tier, and the bottom tier of nodes of the network. Both the manufacturers and the distributors are assumed to be multicriteria decision-makers and concerned not only with profit maximization but also with risk minimization. The risks that manufacturers and distributors face may include, for example, the political risk, the currency risk, or a combination thereof, etc. The bottom tier of decision-makers, namely, the retailers, in turn, are faced with the risk associated with the uncertainty in demands.

We consider a global setting, in which there are $L$ countries, with a typical country denoted by $l, \hat{l}$, or $\bar{l}$ (since we need to distinguish a given country by tier). There are $I$ manufacturers in each country with a typical manufacturer $i$ in country $l$ denoted by $i l$ and associated with node $i l$ in the top tier of nodes in the global supply chain supernetwork (cf. Figure 1). There are, hence, a total of $I L$ manufacturers in the global economy. Also, we consider $J$ distributors in each country with a typical distributor $j$ in country $\hat{l}$ being denoted by $j \hat{l}$ and associated with the second tier node $j \hat{l}$ in the network. There are, thus, a total of $J L$ distributors in the global supply chain network. A typical retailer $k$ in country $\bar{l}$ dealing in currency $h$ is denoted by $k h \bar{l}$ and is associated with the corresponding node in the bottom tier of the network and is, referred to, henceforth, as retailer $k h \bar{l}$, with the understanding that the retailer is associated with a country and transactions in a currency. There are a total of $K H L$ bottom tiered nodes in the global supply chain with $K$ denoting the number of retailers and $H$ the number of currencies.

In order to more clearly understand the processes that drive the dynamics of the proposed system, we assume a homogeneous product economy meaning that the same product is being produced by all manufacturers, shipped to the distributors, who, in turn, distribute the product to the retailers. We allow the manufacturers to transact either physically with the distributors, or directly, in an electronic manner, with the retailers, which reflects the development and popularization of the electronic mode of doing business in today's economy. Therefore, the links connecting the top and the bottom tiers of nodes in Figure 1 represent electronic links. The retailers associated with the bottom tier of nodes of the global supply chain network may not necessarily be physical. They may represent on-line stores or other kinds of virtual retailers. The framework can also handle multiple products, but with a 
concommitant increase in notation.

Note that in Figure 1, there are $H$ distinct links between a manufacturer and a distributor pair. For example, the $h$-th link connecting manufacturer $i l$ with distributor $j \hat{l}$ denotes the possibility of a transaction between this pair of decision-makers in currency $h$. The flow on such a link, hence, is the volume of product transacted between this pair in currency $h$ with the flow denoted by $q_{j h \hat{l}}^{i l}$. We group all such flows for all the manufacturers (in all the countries) and transacting with all the distributors (in all the countries) via the currencies into the column vector $Q^{1} \in R_{+}^{I L J H L}$. In addition, we note that there is a single link connecting each manufacturer $i l$ with each bottom tiered node in the network in Figure 1 and denote the volume of product transacted between $i l$ and retailer $k h \bar{l}$ by $q_{k h \bar{l}}^{i l}$. Such links denote transactions conducted electronically. We group all the product flows between the manufacturers and the retailers into the column vector $Q^{2} \in R_{+}^{I L K H L}$.

Finally, note that the links connecting the distributors with the retailers reflect transactions conducted in a specific currency since the bottom tiered nodes denote retailer/currency / country combinations, and the flow on such an abstract link denotes the volume of product transacted in such a fashion between the manufacturer and retailer pair. Since we allow retailers to be either physical or virtual the link between a distributor and retailer pair may correspond to a physical transaction or to an electronic transaction. We let $q_{k h \bar{l}}^{j \hat{l}}$ denote the volume of product transacted electronically between distributor $j \hat{l}$ in currency $h$ with retailer $k$ in country $\bar{l}$ and group all such flows into the column vector $Q^{3} \in R_{+}^{J L K H L}$.

We now turn to the description of the dynamic adjustment processes which govern the disequilibrium dynamics as the various global supply chain decision-makers adjust their product transactions between the tiers and the prices associated with the different tiers adjust as well. We begin with a discussion of the price dynamics and then describe the dynamics of the product transactions. 


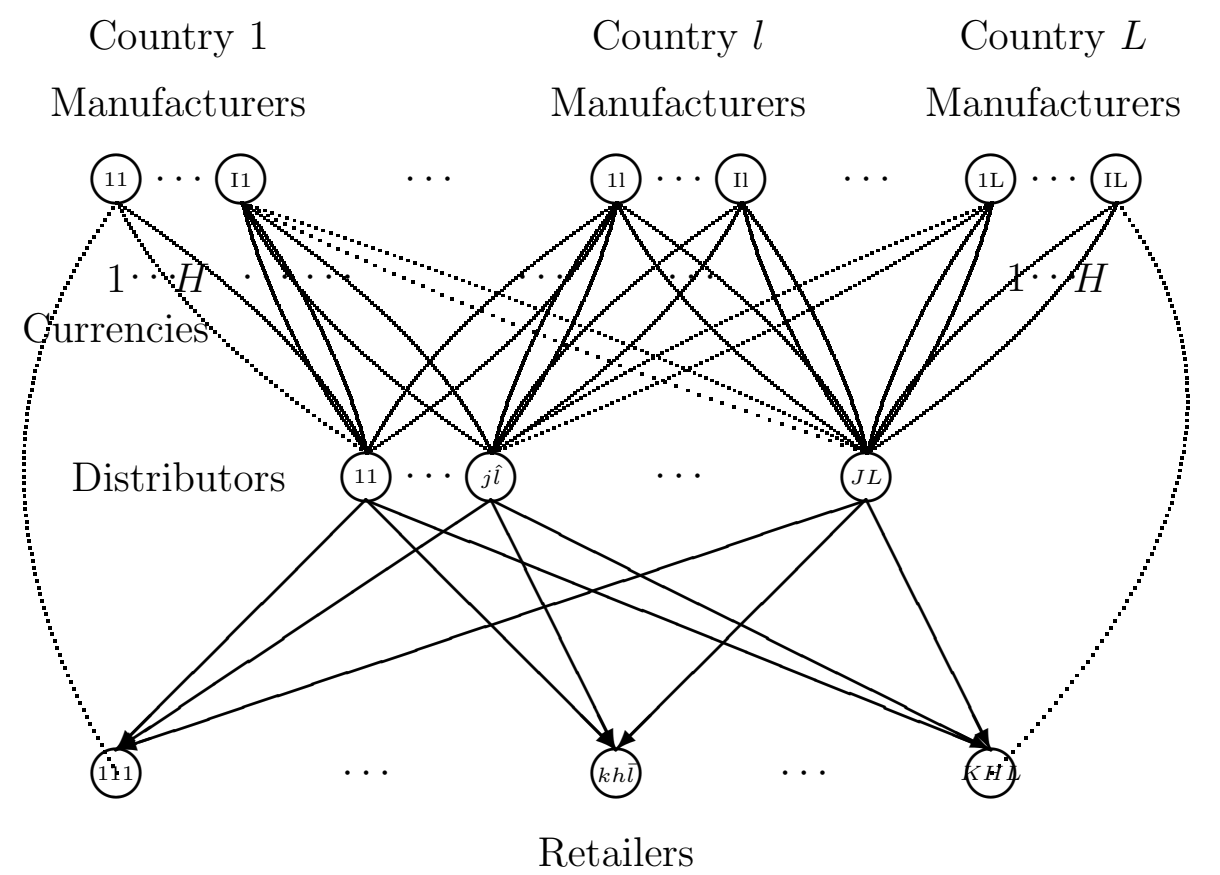

Figure 1: The Structure of the Global Supply Chain Supernetwork

\section{The Retail Price Dynamics}

We begin by describing the dynamics underlying the prices of the product associated with the retailers since the demand for the product drives both the manufacture and the distribution of the product. Let $\rho_{3 k h \bar{l}}$ denote the price of the product associated with retailer $k h \bar{l}$. We assume that $\hat{d}_{k h \bar{l}}\left(\rho_{3 k h \bar{l}}\right)$ is the demand for the product at the price $\rho_{3 k h \bar{l}}$ at retailer $k h \bar{l}$, where $\hat{d}_{k h \bar{l}}\left(\rho_{3 k h \bar{l}}\right)$ is a random variable with density function $F_{k h \bar{l}}\left(x, \rho_{3 k h \bar{l}}\right)$, with $\rho_{3 k h \bar{l}}$ serving as a parameter. As in Dong, Zhang, and Nagurney (2004), we assume that the density function may vary with the retailer price. Let $P_{k h \bar{l}}$ be the probability distribution function of $\hat{d}_{k h \bar{l}}\left(\rho_{3 k h \bar{l}}\right)$, that is, $P_{k h \bar{l}}\left(x, \rho_{3 k h \bar{l}}\right)=P_{k h \bar{l}}\left(\hat{d}_{k h \bar{l}} \leq x\right)=\int_{0}^{x} F_{k h \bar{l}}\left(x, \rho_{3 k h \bar{l}}\right) d x$.

Using the most natural and popular among economists assumption about the forces that influence the behavior of the prices in the market economy (see, for example, Samuelson (1941), Metzler (1945), and Arrow and Hurwicz (1958)), we assume that the rate of change of the price $\rho_{3 k h \bar{l}}$, denoted by $\dot{\rho}_{3 k h \bar{l}}$, is proportional to the difference between the expected demand, where the expected demand is $d_{k h \bar{l}}\left(\rho_{3 k h \bar{l}}\right) \equiv E\left(\hat{d}_{k h \bar{l}}\left(\rho_{3 k h \bar{l}}\right)\right)$, and the total amount transacted with the retailer. Moreover, the rate of change of the price $\rho_{3 k h \bar{l}}$ has the same sign as the difference between the expected demand and the total amount transacted with 
the retailer. Let $\phi_{k h \bar{l}}$ denote the speed of adjustment associated with the price at demand market $k$, currency $h$, and country $\bar{l}$. This term or factor is assumed to be positive.

Hence, we have that for each $k, h, \bar{l}$

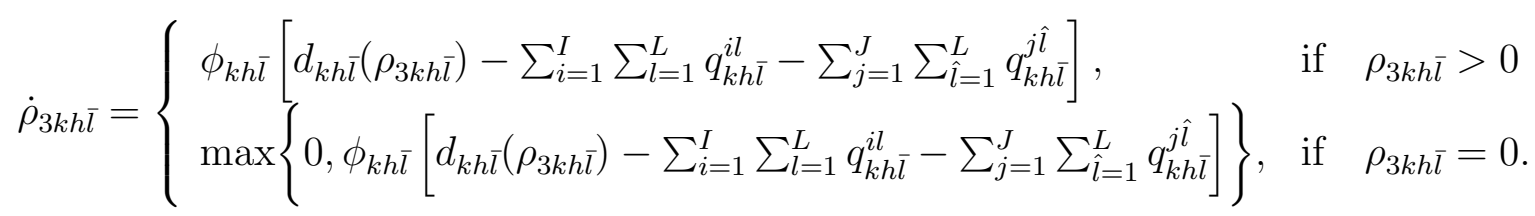

Note that $\phi_{k h \bar{l}}$ may also be interpreted as the sensitivity of the specific demand market in the country and associated with transactions in a particular currency to the changes in the supply and/or demand of the product.

Hence, if the demand for the product at the retailer (at an instant in time) exceeds the amount available, the price of the product at that retailer will increase with the speed $\phi_{k h \bar{l}}$; if the amount available exceeds the demand at the price, then the price at the retailer will decrease with the same speed $\phi_{k h \bar{l}}$. Furthermore, we guarantee that the prices do not become negative.

\section{The Dynamics of the Prices at the Distributors}

The prices charged for the product by various distributors must reflect supply and demand conditions, similar to the case of the retailers. We let $\dot{\gamma}_{j \hat{l}}$ denote the rate of change of the price of the product at distributor $j \hat{l}$. Thus, we assume that the price for the product charged by distributor $j \hat{l}$, associated with the node $j \hat{l}$ in the middle tier of the supply chain network, evolves over time according to:

$$
\dot{\gamma}_{j \hat{l}}=\left\{\begin{array}{lll}
\phi_{j \hat{l}}\left[\sum_{k=1}^{K} \sum_{h=1}^{H} \sum_{\bar{l}=1}^{L} q_{k h \bar{l}}^{j \hat{l}}-\sum_{i=1}^{I} \sum_{l=1}^{L} \sum_{h=1}^{H} q_{j h \hat{l}}^{i l}\right], & \text { if } & \gamma_{j \hat{l}}>0 \\
\max \left\{0, \phi_{j \hat{l}}\left[\sum_{k=1}^{K} \sum_{h=1}^{H} \sum_{\bar{l}=1}^{L} q_{k h \bar{l}}^{j \hat{l}}-\sum_{i=1}^{I} \sum_{l=1}^{L} \sum_{h=1}^{H} q_{j h \hat{l}}^{i l}\right]\right\}, & \text { if } & \gamma_{j \hat{l}}=0 .
\end{array}\right.
$$

and that such dynamics hold for all the distributors $j \hat{l}$. Here, the term $\phi_{j \hat{l}}$ is the speed of adjustment of the price for the product at distributor $j \hat{l}$. This term is also assumed to be positive for all $j \hat{l} s \mathrm{~s}$.

In other words, if the amount of the product desired to be transacted by the retailers (at an instant in time) exceeds that available at the distributor, then the price charged at the distributor will increase with the speed $\phi_{j \hat{l}}$; if the amount available is greater than that desired by the retailer, then the price charged by the distributor will decrease with the same speed $\phi_{j \hat{l}}$. As in the case of the retail prices, we guarantee that the prices charged by the distributors remain nonnegative through the above projection operation. 


\section{Precursors to the Dynamics of the Product Transactions}

Before we describe the dynamics of the product transactions, it is necessary to describe the behavior of the first tier of decision-makers, that is, the manufacturers, and also to introduce some additional notation. Let $q^{i l}$ denote the production output of manufacturer $i l$, which is a nonnegative quantity. We group the production outputs of all manufacturers into the column vector $q \in R_{+}^{I L}$ and assume that each manufacturer $i l$ has a production cost function $f^{i l}$, which can depend, in general, on the entire vector of production outputs, that is,

$$
f^{i l}=f^{i l}(q), \quad \forall i, l .
$$

Therefore, the production cost of a particular manufacturer can depend not only on his production output but also on the production outputs of the other manufacturers. This allows one to model competition.

Let $c_{j h \hat{l}}^{i l}$ denote the transaction cost (which we assume includes the cost of transportation and other expenses) that manufacturer $i l$ is faced with in transacting with distributor $j \hat{l}$ in currency $h$. Let $c_{k h l}^{i l}$, in turn, denote the transaction cost (which also includes the cost of transportation and other expenses) that manufacturer $i l$ is faced with transacting directly with retailer $k$ in currency $h$ and country $\bar{l}$. These transaction costs may depend upon the volume of transactions between each such pair in a particular currency, and their form depends on the type of transaction. They are given, respectively, by:

$$
c_{j h \hat{l}}^{i l}=c_{j h \hat{l}}^{i l}\left(q_{j h \hat{l}}^{i l}\right), \quad \forall i, l, j, h, \hat{l}
$$

and

$$
c_{k h \bar{l}}^{i l}=c_{k h \bar{l}}^{i l}\left(q_{k h \bar{l}}^{i l}\right), \quad \forall i, l, k, h, \bar{l} .
$$

The following conservation of flow equation must hold:

$$
q^{i l}=\sum_{j=1}^{J} \sum_{h=1}^{H} \sum_{\hat{l}=1}^{L} q_{j h \hat{l}}^{i l}+\sum_{k=1}^{K} \sum_{h=1}^{H} \sum_{\bar{l}=1}^{L} q_{k h \bar{l}}^{i l}, \quad \forall i, l .
$$

Thus, according to (5) the amount of the product produced by a manufacturer is equal to the amount transacted with the distributors and with the retailers in the various currencies.

Note that one can express the production cost function of manufacturer $i l$ (cf. (3)) as a function of the vectors $Q^{1}$ and $Q^{2}: f^{i l}\left(Q^{1}, Q^{2}\right)$ and we use this notation, henceforth.

It is assumed that each manufacturer seeks to maximize his profits which is the difference between his revenue and the total costs incurred. The revenue is equal to the product 
of the price and the total quantity transacted with all the distributors and with all the retailers. Since we allow the transactions to take place in different currencies, the prices of the product in different currencies associated with transactions with different tiers of decision-makers may be distinct. Let $\rho_{1 j h \hat{l}}^{i l}$ denote the price associated with the product transacted between manufacturer $i l$ and distributor $j \hat{l}$ in currency $h$, and let $\rho_{1 k h \bar{l}}^{i l}$ denote the price of the product associated with a transaction between manufacturer $i l$ and retailer $k$ in currency $h$ and country $\bar{l}$.

Another assumption to be noted is that all the cost functions are given in the base currency. Considering the fact that the prices are in different currencies and costs are all in the base currency, we need to introduce a device that will bring all the measures to one common denomination. Hence, we introduce the currency exchange rate $e_{h}$, which is the exchange rate of currency $h$ relative to the base currency. The exchange rates are exogenous and fixed in the model whereas all the other prices are endogenous. Given the above, the total revenue of manufacturer $i l$ can be expressed as:

$$
\sum_{j=1}^{J} \sum_{h=1}^{H} \sum_{\hat{l}=1}^{L}\left(\rho_{1 j h \hat{l}}^{i l} \times e_{h}\right) q_{j h \hat{l}}^{i l}+\sum_{k=1}^{K} \sum_{h=1}^{H} \sum_{\bar{l}=1}^{L}\left(\rho_{1 k h \bar{l}}^{i l} \times e_{h}\right) q_{k h \bar{l}}^{i l} .
$$

Consequently, using the conservation of flow equation (5), the production cost functions (3), and the transaction cost functions (4a) and (4b), one can express the profit maximization criterion for manufacturer $i l$ as:

$$
\begin{gathered}
\text { Maximize } \sum_{j=1}^{J} \sum_{h=1}^{H} \sum_{\hat{l}=1}^{L}\left(\rho_{1 j h \hat{l}}^{i l} \times e_{h}\right) q_{j h \hat{l}}^{i l}+\sum_{k=1}^{K} \sum_{h=1}^{H} \sum_{\bar{l}=1}^{L}\left(\rho_{1 k h \bar{l}}^{i l} \times e_{h}\right) q_{k h \bar{l}}^{i l} \\
-f^{i l}\left(Q^{1}, Q^{2}\right)-\sum_{j=1}^{J} \sum_{h=1}^{H} \sum_{\hat{l}=1}^{L} c_{j h \hat{l}}^{i l}\left(q_{j h \hat{l}}^{i l}\right)-\sum_{k=1}^{K} \sum_{h=1}^{H} \sum_{\bar{l}=1}^{L} c_{k h \bar{l}}^{i l}\left(q_{k h \bar{l}}^{i l}\right),
\end{gathered}
$$

subject to: $q_{j h \hat{l}}^{i l} \geq 0$, for all $j, h, \hat{l}$ and $q_{k h \bar{l}}^{i l} \geq 0$, for all $k, h, \bar{l}$.

In addition to the criterion of profit maximization, we also assume that each manufacturer is concerned with risk minimization. Here, for the sake of generality, we assume, as given, a risk function $r^{i l}$, for manufacturer $i$ in country $l$, which is assumed to be continuous and convex and a function of not only the product transactions associated with the particular manufacturer but also of those of other manufacturers. Hence, we assume that

$$
r^{i l}=r^{i l}\left(Q^{1}, Q^{2}\right), \quad \forall i, l .
$$

Note that according to (7), the risk as perceived by a manufacturer is dependent not only upon his product transactions but also on those of other manufacturers. Hence, the second 
criterion of manufacturer $i l$ can be expressed as:

$$
\text { Minimize } r^{i l}\left(Q^{1}, Q^{2}\right)
$$

subject to: $q_{j h \hat{l}}^{i l} \geq 0$, for all $j, h, \hat{l}$ and $q_{k h \bar{l}}^{i l} \geq 0$, for all $k, h, \bar{l}$. The risk function may be distinct for each manufacturer/country combination and can assume whatever form is necessary.

\section{Multicriteria Decision-Making Behavior of the Manufacturers}

Each manufacturer $i l$ considers the risk minimization criterion to be of a certain level of importance, but this level is not necessarily the same for all manufacturers. In order to emphasize this fact, we associate a nonnegative weight $\alpha^{i l}$ with the risk minimization criterion (8), with the weight associated with the profit maximization criterion (6) serving as the numeraire and being set equal to 1 . Thus, we can construct a value function for each manufacturer (cf. Fishburn (1970), Chankong and Haimes (1983), Yu (1985), Keeney and Raiffa (1993)) using a constant additive weight value function. Consequently, letting $U^{i l}$ denote the multicriteria objective function faced by manufacturer $i l$, the decision-making problem for manufacturer $i l$, for $i=1, \ldots, I ; l=1, \ldots, L$, can be transformed into:

$$
\begin{aligned}
& \text { Maximize } U^{i l}=\sum_{j=1}^{J} \sum_{h=1}^{H} \sum_{\hat{l}=1}^{L}\left(\rho_{1 j h \hat{l}}^{i l} \times e_{h}\right) q_{j h \hat{l}}^{i l}+\sum_{k=1}^{K} \sum_{h=1}^{H} \sum_{\bar{l}=1}^{L}\left(\rho_{1 k h \bar{l}}^{i l} \times e_{h}\right) q_{k h \bar{l}}^{i l} \\
& -f^{i l}\left(Q^{1}, Q^{2}\right)-\sum_{j=1}^{J} \sum_{h=1}^{H} \sum_{\hat{l}=1}^{L} c_{j h \hat{l}}^{i l}\left(q_{j h \hat{l}}^{i l}\right)-\sum_{k=1}^{K} \sum_{h=1}^{H} \sum_{\bar{l}=1}^{L} c_{k h \bar{l}}^{i l}\left(q_{k h \bar{l}}^{i l}\right)-\alpha^{i l} r^{i l}\left(Q^{1}, Q^{2}\right),
\end{aligned}
$$

subject to: $q_{j h \hat{l}}^{i l} \geq 0$, for all $j, h, \hat{l}$ and $q_{k h \bar{l}}^{i l} \geq 0$, for all $k, h, \bar{l}$.

It is assumed that the production cost functions and the transaction cost functions for each manufacturer are continuous and convex. It is also assumed that the manufacturers compete according to Nash's concept of noncooperative behavior (cf. Nash $(1950,1951)$ ), which states, in this context, that each manufacturer will determine his optimal production quantity and transactions, given the optimal ones of his competitors. We ignore, for the time being, the nonnegativity constraints on the transaction variables. Let the gradient of manufacturer $i l$ 's multicriteria objective function $U^{i l}$ with respect to the $\hat{q}^{i l}$ variables be denoted by: $\nabla_{\hat{q}^{i l}} U^{i l}=\left(\frac{\partial U^{i l}}{\partial q_{111}^{i l}}, \ldots, \frac{\partial U^{i l}}{\partial q_{J H \hat{L}}^{i l}}\right)$. Note that this gradient represents manufacturer $i l$ 's idealized direction with respect to $\hat{q}^{i l}$, with the $j h \hat{l}$-component of $\nabla_{\hat{q}^{i l}} U^{i l}$ given by:

$$
\left(\rho_{1 j h \hat{l}}^{i l} \times e_{h}-\frac{\partial f^{i l}\left(Q^{1}, Q^{2}\right)}{\partial q_{j h \hat{l}}^{i l}}-\frac{\partial c_{j h \hat{l}}^{i l}\left(q_{j h \hat{l}}^{i l}\right)}{\partial q_{j h \hat{l}}^{i l}}-\alpha^{i l} \frac{\partial r^{i l}\left(Q^{1}, Q^{2}\right)}{\partial q_{j h \hat{l}}^{i l}}\right) .
$$


Similarly, $\nabla_{\bar{q}^{i l}} U^{i l}=\left(\frac{\partial U^{i l}}{\partial q_{111}^{i l}}, \ldots, \frac{\partial U^{i l}}{\partial q_{K H L}^{i l}}\right)$ represents manufacturer $i l$ 's idealized direction with respect to $\bar{q}^{i l}$, with the $k h \bar{l}$-component of $\nabla_{\bar{q}^{i l}} U^{i l}$ being given by:

$$
\left(\rho_{1 k h \bar{l}}^{i l} \times e_{h}-\frac{\partial f^{i l}\left(Q^{1}, Q^{2}\right)}{\partial q_{k h \bar{l}}^{i l}}-\frac{\partial c_{k h \bar{l}}^{i l}\left(q_{k h \bar{l}}^{i l}\right)}{\partial q_{k h \bar{l}}^{i l}}-\alpha^{i l} \frac{\partial r^{i l}\left(Q^{1}, Q^{2}\right)}{\partial q_{k h \bar{l}}^{i l}}\right),
$$

where $\hat{q}^{i l} \equiv\left(q_{111}^{i l}, \ldots, q_{j h \hat{l}}^{i l}, \ldots, q_{J H L}^{i l}\right)$ and $\bar{q}^{i l} \equiv\left(q_{111}^{i l}, \ldots, q_{k h \bar{l}}^{i l}, \ldots, q_{K H L}^{i l}\right)$.

Later, we include the nonnegativity assumption on the product transactions, when we construct the projected dynamical system that captures the dynamics of all the product transactions and prices.

\section{Multicriteria Decision-Making Behavior of the Distributors}

The distributors are involved in transactions with both the manufacturers (since they need to obtain the product for distribution) and with the retailers, who sell the product to the consumers. As was mentioned earlier, since we are dealing with a global supply chain, the distributors, similar to the manufacturers, are allowed to transact in any of the $H$ available currencies.

A distributor $j \hat{l}$ is faced with certain expenses, which may include, for example, the loading/unloading costs, the storage costs, etc., associated with handling the product. We refer collectively to such costs as a handling cost and denote it by $c_{j \hat{l}}$. In a simple situation, one might have that

$$
c_{j \hat{l}}=c_{j \hat{l}}\left(\sum_{i=1}^{I} \sum_{l=1}^{L} \sum_{h=1}^{H} q_{j h \hat{l}}^{i l}, \sum_{k=1}^{K} \sum_{h=1}^{H} \sum_{\bar{l}=1}^{L} q_{k h \bar{l}}^{j \hat{l}}\right),
$$

that is, the handling cost of a distributor is a function of how much of the product he has obtained and how much of the product he has transacted with the various retailers. However, for the sake of generality, and to enhance the modeling of competition, we allow the function to depend also on the amount of the product acquired and transacted by other distributors. Hence, we assume that, for all $j \hat{l}$ :

$$
c_{j \hat{l}}=c_{j \hat{l}}\left(Q^{1}, Q^{3}\right)
$$

Let $\rho_{2 k h \bar{l}}^{j \hat{l}}$ denote the price in currency $h$ associated with the transaction between distributor $j \hat{l}$ and retailer $k h \bar{l}$. The total amount of revenue the distributor obtains from his transactions is equal to the sum of the price (transformed into the base currency) and the amount of the product transacted with the various retailers in the distinct countries and 
currencies. Indeed, since transactions can be made in distinct currencies and with different retailers, who, in fact, may even be virtual, the total revenue of distributor $j \hat{l}$ can be expressed in the base currency as follows:

$$
\sum_{k=1}^{K} \sum_{h=1}^{H} \sum_{\bar{l}=1}^{L}\left(\rho_{2 k h \bar{l}}^{j \hat{l}} \times e_{h}\right) q_{k h \bar{l}}^{j \hat{l}} .
$$

The profit maximization problem for distributor $j \hat{l}$ can be expressed as:

$$
\text { Maximize } \sum_{k=1}^{K} \sum_{h=1}^{H} \sum_{\bar{l}=1}^{L}\left(\rho_{2 k h \bar{l}}^{j \hat{l}} \times e_{h}\right) q_{k h \bar{l}}^{j \hat{l}}-c_{j \hat{l}}\left(Q^{1}, Q^{3}\right)-\sum_{i=1}^{I} \sum_{l=1}^{L} \sum_{h=1}^{H}\left(\rho_{1 j h \hat{l}}^{i l} \times e_{h}\right) q_{j h \hat{l}}^{i l}
$$

subject to:

$$
\sum_{k=1}^{K} \sum_{h=1}^{H} \sum_{\bar{l}=1}^{L} q_{k h \bar{l}}^{j \hat{l}} \leq \sum_{i=1}^{I} \sum_{l=1}^{L} \sum_{h=1}^{H} q_{j h \hat{l}}^{i l}
$$

and the nonnegativity assumptions: $q_{j h \hat{l}}^{i l} \geq 0$, for all $i, l$ and $q_{k h \bar{l}}^{j \hat{l}} \geq 0$, for all $k, h, \bar{l}$.

Constraint (15) states that a distributor in a country cannot transact more of the product than he has obtained from the various manufacturers.

In addition, distributor $j \hat{l}$ is faced with his own perception of risk associated with obtaining and shipping the product, which we assume is incorporated into a continuous risk function of a general form given by:

$$
r^{j \hat{l}}=r^{j \hat{l}}\left(Q^{1}, Q^{3}\right), \quad \forall j, \hat{l} .
$$

Note that we allow the risk function of a particular distributor to depend on the transactions to and from all the distributors in the supply chain network economy.

We assume also that each distributor associates a weight of 1 with the profit criterion (14) and a weight of $\beta^{j \hat{l}}$ with his risk level. Therefore, the multicriteria decision-making problem for distributor $j \hat{l} ; j=1, \ldots, J ; \hat{l}=1, \ldots, L$, faced now with a multicriteria objective function denoted by $U^{j \hat{l}}$ can be transformed directly into the optimization problem:

$$
\begin{gathered}
\text { Maximize } U^{j \hat{l}}=\sum_{k=1}^{K} \sum_{h=1}^{H} \sum_{\bar{l}=1}^{L}\left(\rho_{2 k h \bar{l}}^{j \hat{l}} \times e_{h}\right) q_{k h \bar{l}}^{j \hat{l}}-c_{j \hat{l}}\left(Q^{1}, Q^{3}\right)-\sum_{i=1}^{I} \sum_{l=1}^{L} \sum_{h=1}^{H}\left(\rho_{1 j h \hat{l}}^{i l} \times e_{h}\right) q_{j h \hat{l}}^{i l} \\
-\beta^{j \hat{l}} r^{j \hat{l}}\left(Q^{1}, Q^{3}\right)
\end{gathered}
$$

subject to:

$$
\sum_{k=1}^{K} \sum_{h=1}^{H} \sum_{\bar{l}=1}^{L} q_{k h \bar{l}}^{j \hat{l}} \leq \sum_{i=1}^{I} \sum_{l=1}^{L} \sum_{h=1}^{H} q_{j h \hat{l}}^{i l}
$$


and the nonnegativity constraints: $q_{j h \hat{l}}^{i l} \geq 0$, and $q_{k h \bar{l}}^{j \hat{l}} \geq 0$, for all $i, h, k, l$, and $\bar{l}$.

Objective function (17) represents a value function for distributor $j \hat{l}$ with $\beta^{j \hat{l}}$ having the interpretation as a conversion rate in dollar value.

For simplicity of notation, we let $q_{j \hat{l}}=\left(q_{j 1 \hat{l}}^{11}, \ldots, q_{j h \hat{l}}^{i l}, \ldots, q_{j H \hat{l}}^{I L}\right)$ and $q^{j \hat{l}}=\left(q_{111}^{j \hat{l}}, \ldots, q_{k h \bar{l}}^{j \hat{l}}, \ldots, q_{K H L}^{j \hat{l}}\right)$.

Ignoring, for the time being, the nonnegativity constraints (as was done above for the manufacturers), and constraint $(18), \nabla_{q_{j \hat{l}}} U^{j \hat{l}}=\left(\frac{\partial U^{j \hat{l}}}{\partial q_{j 1 \hat{l}}^{1 L}}, \ldots, \frac{\partial U^{j \hat{l}}}{\partial q_{j H \hat{l}}^{I L}}\right)$ represents distributor $j \hat{l}^{\prime} \mathrm{s}$ idealized direction in terms of $q_{j \hat{l}}$, where component $i l h$ is given by:

$$
\left(\gamma_{j \hat{l}}-\frac{\partial c_{j \hat{l}}\left(Q^{1}, Q^{3}\right)}{\partial q_{j h \hat{l}}^{i l}}-\beta^{j \hat{l}} \frac{\partial r^{j \hat{l}}\left(Q^{1}, Q^{3}\right)}{\partial q_{j h \hat{l}}^{i l}}-\rho_{1 j h \hat{l}}^{i l} \times e_{h}\right)
$$

whereas $\nabla_{q^{j} \hat{l}} U^{j \hat{l}}=\left(\frac{\partial U^{j \hat{l}}}{\partial q_{111}^{j \hat{l}}}, \ldots, \frac{\partial U^{j \hat{l}}}{\partial q_{K H L}^{j \hat{l}}}\right)$ represents distributor $j \hat{l}$ 's idealized direction in terms of the $q^{j \hat{l}}$ variables, with component $k h \bar{l}$ given by:

$$
\left(\rho_{2 k h \bar{l}}^{j \hat{l}} \times e_{h}-\frac{\partial c_{j \hat{l}}\left(Q^{1}, Q^{3}\right)}{\partial q_{k h \bar{l}}^{j \hat{l}}}-\beta^{j \hat{l}} \frac{\partial r^{j \hat{l}}\left(Q^{1}, Q^{3}\right)}{\partial q_{k h \bar{l}}^{j \hat{l}}}-\gamma_{j \hat{l}}\right)
$$

However, manufacturer $i l$ and distributor $j \hat{l}$ must agree on the volume of $q_{j h \hat{l}}^{i l}$ in order for the transaction to actually take place in currency $h$. Therefore, direction (10) of manufacturer $i l$ must coincide with direction (19) of distributor $j \hat{l}$ transacting in currency $h$, yielding, after algebraic simplification, a combined direction, which signifies cooperation, given by:

$$
\left(\gamma_{j \hat{l}}-\frac{\partial f^{i l}\left(Q^{1}, Q^{2}\right)}{\partial q_{j h \hat{l}}^{i l}}-\frac{\partial c_{j h \hat{l}}^{i l}\left(q_{j h \hat{l}}^{i l}\right)}{\partial q_{j h \hat{l}}^{i l}}-\frac{\partial c_{j \hat{l}}\left(Q^{1}, Q^{3}\right)}{\partial q_{j h \hat{l}}^{i l}}-\alpha^{i l} \frac{\partial r^{i l}\left(Q^{1}, Q^{2}\right)}{\partial q_{j h \hat{l}}^{i l}}-\beta^{j \hat{l}} \frac{\partial r^{j \hat{l}}\left(Q^{1}, Q^{3}\right)}{\partial q_{j h \hat{l}}^{i l}}\right) .
$$

Moreover, (21) must hold for all $i, l$ and $j, \hat{l}, h$ for the supply chain to allow for links between the top two tiers of nodes.

We note that such cooperation needs to occur not only in supply chains but in other multitiered networks such as financial networks with intermediation (cf. Nagurney and Ke (2003) and Nagurney and Cruz (2003)).

\section{Optimizing Behavior of the Retailers with Uncertain Demands}

The retailers, in turn, are faced with the decision of how much product to acquire from the distributors and from the manufacturers (via the Internet) in order to satisfy the random 
demand while still seeking to maximize profits. A retailer $k h \bar{l}$ has his own handling cost, which may include, for example, various expenses related to the display and storage of the product. We denote this cost by $c_{k h \bar{l}}$ and, in the simplest case, we would have that $c_{k h \bar{l}}\left(s_{k h \bar{l}}\right)$, where $s_{k h \bar{l}}=\sum_{i=1}^{I} \sum_{l=1}^{L} q_{k h \bar{l}}^{i l}+\sum_{j=1}^{J} \sum_{\bar{l}=1}^{L} q_{k h \bar{l}}^{j \hat{l}}$, that is, the holding cost of a retailer is a function of how much of the product he has obtained from transactions with the various manufacturers and the various distributors. However, in general, this cost may be affected by the amounts of the product held by other retailers. Therefore, we write:

$$
c_{k h \bar{l}}=c_{k h \bar{l}}\left(Q^{2}, Q^{3}\right), \quad \forall k, h, \bar{l} .
$$

Recall that $\rho_{3 k h \bar{l}}$ denotes the price of the product associated with retailer $k h \bar{l}$. Also recall that $\hat{d}_{k h \bar{l}}\left(\rho_{3 k h \bar{l}}\right)$ is the demand for the product at the price $\rho_{3 k h \bar{l}}$ at retailer $k h \bar{l}$, where $\hat{d}_{k h \bar{l}}\left(\rho_{3 k h \bar{l}}\right)$ is a random variable with a density function of $F_{k h \bar{l}}\left(x, \rho_{3 k h \bar{l}}\right)$.

Since the demand is random and a retailer does not know how much he will sell beforehand, the actual sale of the product at retailer $k h \bar{l}$ cannot exceed $\min \left\{s_{k h \bar{l}}, \hat{d}_{k h \bar{l}}\right\}$. Let

$$
\Delta_{k h \bar{l}}^{+} \equiv \max \left\{0, s_{k h \bar{l}}-\hat{d}_{k h \bar{l}}\right\}
$$

and

$$
\Delta_{k h \bar{l}}^{-} \equiv \max \left\{0, \hat{d}_{k h \bar{l}}-s_{k h \bar{l}}\right\}
$$

where $\Delta_{k h \bar{l}}^{+}$is a random variable representing the excess supply (inventory), whereas $\Delta_{k h \bar{l}}^{-}$is a random variable representing the excess demand (shortage).

Note that the expected values of excess supply and excess demand of retailer $k h \bar{l}$ are scalar functions of $s_{k h \bar{l}}$ and $\rho_{3 k h \bar{l}}$. In particular, let $\pi_{k h \bar{l}}^{+}$and $\pi_{k h \bar{l}}^{-}$denote, respectively, the expected values $E\left(\Delta_{k h \bar{l}}^{+}\right)$and $E\left(\Delta_{k h \bar{l}}^{-}\right)$, that is,

$$
\pi_{k h \bar{l}}^{+}\left(s_{k h \bar{l}}, \rho_{3 k h \bar{l}}\right) \equiv E\left(\Delta_{k h \bar{l}}^{+}\right)=\int_{0}^{s_{k h \bar{l}}}\left(s_{k h \bar{l}}-x\right) F_{k h \bar{l}}\left(x, \rho_{3 k h \bar{l}}\right) d x
$$

and

$$
\pi_{k h \bar{l}}^{-}\left(s_{k h \bar{l}}, \rho_{3 k h \bar{l}}\right) \equiv E\left(\Delta_{k h \bar{l}}^{-}\right)=\int_{s_{k h \bar{l}}}^{\infty}\left(x-s_{k h \bar{l}}\right) F_{k h \bar{l}}\left(x, \rho_{3 k h \bar{l}}\right) d x
$$

Assume now that retailer $k h \bar{l}$ is faced with certain penalties for having an excess or shortage in regards to the supply. Let $\lambda_{k h \bar{l}}^{+} \geq 0$ denote the inventory carrying cost at retail outlet $k h \bar{l}$, and let $\lambda_{k h \bar{l}}^{-} \geq 0$ denote the unit penalty of having excess demand at outlet $k h \bar{l}$. Then the expected total penalty of retailer $k h \bar{l}$ can be expressed as:

$$
E\left(\lambda_{k h \bar{l}}^{+} \Delta_{k h \bar{l}}^{+}+\lambda_{k h \bar{l}}^{-} \Delta_{k h \bar{l}}^{-}\right)=\lambda_{k h \bar{l}}^{+} \pi_{k h \bar{l}}^{+}\left(s_{k h \bar{l}}, \rho_{3 k h \bar{l}}\right)+\lambda_{k h \bar{l}}^{-} \pi_{k h \bar{l}}^{-}\left(s_{k h \bar{l}}, \rho_{3 k h \bar{l}}\right) .
$$


Assuming also profit-maximizing behavior of the retailers, one can state the following optimization problem for retailer $k h \bar{l}$ :

$$
\begin{gathered}
\text { Maximize } \quad E\left(\left(\rho_{3 k h \bar{l}} \times e_{h}\right) \cdot \min \left\{s_{k h \bar{l}}, \hat{d}_{k h \bar{l}}\right\}\right)-E\left(\lambda_{k h \bar{l}}^{+} \Delta_{k h \bar{l}}^{+}+\lambda_{k h \bar{l}}^{-} \Delta_{k h \bar{l}}^{-}\right) \\
-c_{k h \bar{l}}\left(Q^{2}, Q^{3}\right)-\sum_{i=1}^{I} \sum_{l=1}^{L}\left(\rho_{1 k h \bar{l}}^{i l} \times e_{h}\right) q_{k h \bar{l}}^{i l}-\sum_{j=1}^{J} \sum_{\hat{l}=1}^{L}\left(\rho_{2 k h \bar{l}}^{j \hat{l}} \times e_{h}\right) q_{k h \bar{l}}^{j \hat{l}}
\end{gathered}
$$

subject to: $q_{k h \bar{l}}^{i l} \geq 0, q_{k h \bar{l}}^{j \hat{l}} \geq 0$, for all $i, l, j, \hat{l}$.

Applying now the definitions of $\Delta_{k h \bar{l}}^{+}$and $\Delta_{k h \bar{l}}^{-}$, we know that $\min \left\{s_{k h \bar{l}}, \hat{d}_{k h \bar{l}}\right\}=\hat{d}_{k h \bar{l}}-\Delta_{k h \bar{l}}^{-}$, we can express the objective function (27) (and denoted by $U^{k h \bar{l}}$ ) as

$$
\begin{aligned}
& \text { Maximize } \quad U^{k h \bar{l}}=\left(\rho_{3 k h \bar{l}} \times e_{h}\right) d_{k h \bar{l}}\left(\rho_{3 k h \bar{l}}\right)-\left(\rho_{3 k h \bar{l}} \times e_{h}+\lambda_{k h \bar{l}}^{-}\right) \pi_{k h \bar{l}}^{-}\left(s_{k h \bar{l}}, \rho_{3 k h \bar{l}}\right)-\lambda_{k h \bar{l}}^{+} \pi_{k h \bar{l}}^{+}\left(s_{k h \bar{l}}, \rho_{3 k h \bar{l}}\right) \\
& -c_{k h \bar{l}}\left(Q^{2}, Q^{3}\right)-\sum_{i=1}^{I} \sum_{l=1}^{L}\left(\rho_{1 k h \bar{l}}^{i l} \times e_{h}\right) q_{k h \bar{l}}^{i l}-\sum_{j=1}^{J} \sum_{\hat{l}=1}^{L}\left(\rho_{2 k h \bar{l}}^{j \hat{l}} \times e_{h}\right) q_{k h \bar{l}}^{j \hat{l}},
\end{aligned}
$$

where $d_{k h \bar{l}}\left(\rho_{3 k h \bar{l}}\right) \equiv E\left(\hat{d}_{k h \bar{l}}\right)$ is a scalar function of $\rho_{3 k h \bar{l}}$.

At this point it is necessary to note the following relationships:

$$
\begin{gathered}
\frac{\partial \pi_{k h \bar{l}}^{+}\left(s_{k h \bar{l}}, \rho_{3 k h \bar{l}}\right)}{\partial q_{k h \bar{l}}^{i l}}=\frac{\partial \pi_{k h \bar{l}}^{+}\left(s_{k h \bar{l}}, \rho_{3 k h \bar{l}}\right)}{\partial q_{k h \bar{l}}^{j \hat{l}}}=P_{k h \bar{l}}\left(s_{k h \bar{l}}, \rho_{3 k h \bar{l}}\right)=P_{k h \bar{l}}\left(\sum_{i=1}^{I} \sum_{l=1}^{L} q_{k h \bar{l}}^{i l}+\sum_{j=1}^{J} \sum_{\hat{l}=1}^{L} q_{k h \bar{l}}^{j \hat{l}}, \rho_{3 k h \bar{l}}\right), \\
\frac{\partial \pi_{k h \bar{l}}^{-}\left(s_{k h \bar{l}}, \rho_{3 k h \bar{l}}\right)}{\partial q_{k h \bar{l}}^{i l}}=\frac{\partial \pi_{k h \bar{l}}^{-}\left(s_{k h \bar{l}}, \rho_{3 k h \bar{l}}\right)}{\partial q_{k h \bar{l}}^{j \hat{l}}}=P_{k h \bar{l}}\left(s_{k h \bar{l}}, \rho_{3 k h \bar{l}}\right)-1 \\
=P_{k h \bar{l}}\left(\sum_{i=1}^{I} \sum_{l=1}^{L} q_{k h \bar{l}}^{i l}+\sum_{j=1}^{J} \sum_{\hat{l}=1}^{L} q_{k h \bar{l}}^{j \hat{l}}, \rho_{3 k h \bar{l}}\right)-1 .
\end{gathered}
$$

Similar to the case of the manufacturers, it is assumed that all the functions in (28) are continuous. It is also assumed that the retailers compete according to Nash's concept of noncooperative behavior, which states, in this context, that each retailer will determine his optimal transactions, given the optimal ones of his competitors. Moreover, (as we have done previously for the manufacturers and the distributors) if we ignore, for the time being that the product transactions must be nonnegative, then retailer $k h \bar{l}$ is faced with an unconstrained utility maximization problem, where $\nabla_{\hat{q}_{k h \bar{l}}} U^{k h \bar{l}}=\left(\frac{\partial U^{k h \bar{l}}}{\partial q_{k h \bar{l}}^{11}}, \ldots, \frac{\partial U^{k h \bar{l}}}{\partial q_{k h \bar{l}}^{J L}}\right)$ represents retailer $k h \bar{l}$ 's idealized direction with respect to the $\hat{q}_{k h \bar{l}}$ variables, with the $j \hat{l}$-component of $\nabla_{\hat{q}_{k h l}} U^{k h \bar{l}}$ being given by:

$$
\left(\left(\lambda_{k h \bar{l}}^{-}+\rho_{3 k h \bar{l}} \times e_{h}\right)\left(1-P_{k h \bar{l}}\left(s_{k h \bar{l}}, \rho_{3 k h \bar{l}}\right)\right)-\lambda_{k h \bar{l}}^{+} P_{k h \bar{l}}\left(s_{k h \bar{l}}, \rho_{3 k h \bar{l}}\right)-\frac{\partial c_{k h \bar{l}}\left(Q^{2}, Q^{3}\right)}{\partial q_{k h \bar{l}}^{j \hat{l}}}-\rho_{2 k h \bar{l}}^{j \hat{l}} \times e_{h}\right),
$$


whereas $\nabla_{\bar{q}_{k h \bar{l}}} U^{k h \bar{l}}=\left(\frac{\partial U^{k h \bar{l}}}{\partial q_{k h \bar{l}}^{11}}, \ldots, \frac{\partial U^{k h \bar{l}}}{\partial q_{k h \bar{l}}^{I L}}\right)$ represents retailer $k h \bar{l}$ 's idealized direction with respect to the $\bar{q}_{k h \bar{l}}$ variables, with the $i l$-component of $\nabla_{\bar{q}_{k h \bar{l}}} U^{k h \bar{l}}$ being given by:

$\left(\left(\lambda_{k h \bar{l}}^{-}+\rho_{3 k h \bar{l}} \times e_{h}\right)\left(1-P_{k h \bar{l}}\left(s_{k h \bar{l}}, \rho_{3 k h \bar{l}}\right)\right)-\lambda_{k h \bar{l}}^{+} P_{k h \bar{l}}\left(s_{k h \bar{l}}, \rho_{3 k h \bar{l}}\right)-\frac{\partial c_{k h \bar{l}}\left(Q^{2}, Q^{3}\right)}{\partial q_{k h \bar{l}}^{i l}}-\rho_{1 k h \bar{l}}^{i l} \times e_{h}\right)$,

where $\hat{q}_{k h \bar{l}} \equiv\left(q_{k h \bar{l}}^{11}, \ldots, q_{k h \bar{l}}^{i l}, \ldots, q_{k h \bar{l}}^{I L}\right)$ and $\bar{q}_{k h \bar{l}} \equiv\left(q_{k h \bar{l}}^{11}, \ldots, q_{k h \bar{l}}^{j \hat{l}}, \ldots, q_{k h \bar{l}}^{J L}\right)$.

Note that in order for the transaction between distributor $j \hat{l}$ and retailer $k h \bar{l}$ to take place, they must agree in terms of the $q_{k h \bar{l}}^{j \hat{l}}$. Therefore, directions (20) and (31) must coincide, with the summation of the two yielding a combined direction given by, after algebraic simplification:

$$
\begin{gathered}
\left(\left(\lambda_{k h \bar{l}}^{-}+\rho_{3 k h \bar{l}} \times e_{h}\right)\left(1-P_{k h \bar{l}}\left(s_{k h \bar{l}}, \rho_{3 k h \bar{l}}\right)\right)-\lambda_{k h \bar{l}}^{+} P_{k h \bar{l}}\left(s_{k h \bar{l}}, \rho_{3 k h \bar{l}}\right)-\frac{\partial c_{j \hat{l}}\left(Q^{1}, Q^{3}\right)}{\partial q_{k h \bar{l}}^{j \hat{l}}}\right. \\
\left.-\frac{\partial c_{k h \bar{l}}\left(Q^{2}, Q^{3}\right)}{\partial q_{k h \bar{l}}^{j \hat{l}}}-\beta^{j \hat{l}} \frac{\partial r^{j \hat{l}}\left(Q^{1}, Q^{3}\right)}{\partial q_{k h \bar{l}}^{j \hat{l}}}-\gamma_{j \hat{l}}\right) .
\end{gathered}
$$

Similarly, for the transaction between a retailer $k h \bar{l}$ and a manufacturer $i l$ to take place, both must agree on the $q_{k h \bar{l}}^{i l}$. Therefore, directions (32) and (11) must coincide as well, with the summation of the two yielding also a combined direction, which, after algebraic simplifications, reduces to:

$$
\begin{gathered}
\left(\left(\lambda_{k h \bar{l}}^{-}+\rho_{3 k h \bar{l}} \times e_{h}\right)\left(1-P_{k h \bar{l}}\left(s_{k h \bar{l}}, \rho_{3 k h \bar{l}}\right)\right)-\frac{\partial f^{i l}\left(Q^{1}, Q^{2}\right)}{\partial q_{k h \bar{l}}^{i l}}-\frac{\partial c_{k h \bar{l}}^{i l}\left(q_{k h \bar{l}}^{i l}\right)}{\partial q_{k h \bar{l}}^{i l}}\right. \\
\left.-\frac{\partial c_{k h \bar{l}}\left(Q^{2}, Q^{3}\right)}{\partial q_{k h \bar{l}}^{i l}}-\alpha^{i l} \frac{\partial r^{i l}\left(Q^{1}, Q^{2}\right)}{\partial q_{k h \bar{l}}^{i l}}-\lambda_{k h \bar{l}}^{+} P_{k h \bar{l}}\left(s_{k h \bar{l}}, \rho_{3 k h \bar{l}}\right)\right) .
\end{gathered}
$$

We are now ready to express the dynamics of the transactions between the tiers of decision-makers in the supply chain supernetwork. Moreover, we now explicitly include the nonnegativity constraints associated with the product transactions and note that (18) has actually been subsumed within the distributor price dynamics given by (2). We then provide a unified expression in the form of a projected dynamical system for the dynamic evolution of all the product transactions and the product prices simultaneously.

The adjustment assumptions to be introduced were suggested by a number of prominent economists (see, for example, Fisher (1961)) and are based on the following reasoning. The rate of change in the amount of good produced/transacted by a particular decision-maker is 
proportional to his marginal profit and has the same sign. In other words, a typical decisionmaker will try to increase his output for as long as the marginal profit is nonnegative, and will try to decrease his output for as long as the marginal profit is negative until the output is zero (that is, hits the boundary for the variable). As was stated earlier, for any transaction to take place, the decision-makers involved have to agree on the amount to be transacted. Thus, the marginal profit of the transaction is represented by the combined direction. Therefore, we can expect that the rate of change in the amount of product transacted between two particular decision-makers is proportional to their combined optimal direction.

\section{The Dynamics of the Product Transactions between Manufacturers and Distrib- utors}

The rate of change in the amount of product $q_{j h \hat{l}}^{i l}$, transacted between manufacturer $i l$ and distributor $j \hat{l}$, and denoted by $\dot{q}_{j h \hat{l}}^{i l}$, is proportional to the combined direction (21) with the coefficient of proportionality $\phi_{j h \hat{l}}^{i l}>0 \quad \forall i, l, j, h, \hat{l}$. Guaranteeing now that the product transactions do not assume negative quantities, we get the following dynamics for the transactions between each manufacturer $i l$ and every distributor $j \hat{l}$ transacting in currency $h$, for all $h$ :

$$
\dot{q}_{j h \hat{l}}^{i l}= \begin{cases}\phi_{j h \hat{l}}^{i l}\left(\gamma_{j \hat{l}}-\frac{\partial f^{i l}\left(Q^{1}, Q^{2}\right)}{\partial q_{j h \hat{l}}^{i l}}-\frac{\partial c_{j h \hat{l}}^{i l}\left(q_{j h \hat{l}}^{i l}\right)}{\partial q_{j h \hat{l}}^{i l}}-\frac{\partial c_{j \hat{l}}\left(Q^{1}, Q^{3}\right)}{\partial q_{j h \hat{\imath}}^{i l}}\right. & \\ \left.-\alpha^{i l} \frac{\partial r^{i l}\left(Q^{1}, Q^{2}\right)}{\partial q_{j h \hat{l}}^{i l}}-\beta^{j \hat{l}} \frac{\partial r^{j \hat{l}}\left(Q^{1}, Q^{3}\right)}{\partial q_{j h \hat{l}}^{i l}}\right), & \text { if } \quad q_{j h \hat{l}}^{i l}>0 \\ \max \left\{0, \phi_{j h \hat{l}}^{i l}\left(\gamma_{j \hat{l}}-\frac{\partial f^{i l}\left(Q^{1}, Q^{2}\right)}{\partial q_{j h \hat{l}}^{i l}}-\frac{\partial c_{j h \hat{\imath}}^{i l}\left(q_{h h \hat{l}}^{i l}\right)}{\partial q_{j h \hat{l}}^{i l}}-\frac{\partial c_{j \hat{l}}\left(Q^{1}, Q^{3}\right)}{\partial q_{j h \hat{l}}^{i l}}\right.\right. & \\ \left.\left.-\alpha^{i l} \frac{\partial r^{i l}\left(Q^{1}, Q^{2}\right)}{\partial q_{j h \hat{l}}^{i l}}-\beta^{j \hat{l}} \frac{\partial r^{j \hat{l}}\left(Q^{1}, Q^{3}\right)}{\partial q_{j h \hat{l}}^{i l}}\right)\right\}, & \text { if } q_{j h \hat{l}}^{i l}=0 .\end{cases}
$$

Here $\phi_{j h \hat{l}}^{i l}$ denotes the (positive) speed of adjustment associated with the particular transaction.

The expression above states that whenever there is a positive difference between the shadow price of the distributor and the aggregated marginal costs and risks associated with a transaction, the amount of "flow" on that particular link will increase with the speed $\phi_{j h \hat{l}}^{i l}$. Otherwise, the amount of flow will either decrease with the same speed or remain the same.

\section{The Dynamics of the Product Transactions between Manufacturers and Retailers}

In a similar manner, we can derive the dynamics associated with the transactions between the manufacturers and the retailers. Recall that such transactions take place on the Internet, 
and that the combined direction of a transaction between manufacturer $i l$ and retailer $k h \bar{l}$ is given by expression (34). Indeed, we may immediately write, requiring now that these transactions must also yield nonnegative values, that for all manufacturers $i l$ and all retailers $k h \bar{l}$, with $\dot{q}_{k h \bar{l}}^{i l}$ denoting the rate of change of the transactions between manufacturer $i l$ and retailer $k h \bar{l}$ :

$$
\dot{q}_{k h \bar{l}}^{i l}=\left\{\begin{array}{l}
\phi_{k h \bar{l}}^{i l}\left(\left(\lambda_{k h \bar{l}}^{-}+\rho_{3 k h \bar{l}} \times e_{h}\right)\left(1-P_{k h \bar{l}}\left(s_{k h \bar{l}}, \rho_{3 k h \bar{l}}\right)\right)-\frac{\partial f^{i l}\left(Q^{1}, Q^{2}\right)}{\partial q_{k h \bar{l}}^{i l}}\right. \\
\left.-\frac{\partial c_{k h \bar{l}}^{i l}\left(q_{k h \bar{l}}^{i l}\right)}{\partial q_{k h \bar{l}}^{i l}}-\frac{\partial c_{k h \bar{l}}\left(Q^{2}, Q^{3}\right)}{\partial q_{k h \bar{l}}^{l i}}-\alpha^{i l} \frac{\partial r^{i l}\left(Q^{1}, Q^{2}\right)}{\partial q_{k h \bar{l}}^{i l}}-\lambda_{k h \bar{l}}^{+} P_{k h \bar{l}}\left(s_{k h \bar{l}}, \rho_{3 k h \bar{l}}\right)\right), \\
\max \left\{0, \phi_{k h \bar{l}}^{i l}\left(\left(\lambda_{k h \bar{l}}^{-}+\rho_{3 k h \bar{l}} \times e_{h}\right)\left(1-P_{k h \bar{l}}\left(s_{k h \bar{l}}, \rho_{3 k h \bar{l}}\right)\right)-\frac{\partial f^{i l}\left(Q^{1}, Q^{2}\right)}{\partial q_{k h \bar{l}}^{i l}}\right.\right. \\
\left.\left.-\frac{\partial c_{k h \bar{l}}^{i l}\left(q_{h h \bar{l}}^{i l}\right)}{\partial q_{k h \bar{l}}^{i l}}-\frac{\partial c_{k h \bar{l}}\left(Q^{2}, Q^{3}\right)}{\partial q_{k h \bar{l}}^{i l}}-\alpha^{i l} \frac{\partial r^{i l}\left(Q^{1}, Q^{2}\right)}{\partial q_{k h \bar{l}}^{i l}}-\lambda_{k h \bar{l}}^{+} P_{k h \bar{l}}\left(s_{k h \bar{l}}, \rho_{3 k h \bar{l}}\right)\right)\right\}, \\
\text { if } \quad q_{k h \bar{l}}^{i l}=0 .
\end{array}\right.
$$

Here, similar to (34), the term $\phi_{k h \bar{l}}^{i l}$ for all $i, l, k, h, \bar{l}$, which is assumed to be positive, is a speed of adjustment.

Hence, (36) expresses that the volume of transactions between a manufacturer and retailer pair will increase if the, what may be termed, generalized price associated with the product in that currency at the retailer exceeds the various marginal costs and risks (with the probabilities associated with excess supply and demand incorporated into the computations).

\section{The Dynamics of the Product Transactions between Distributors and Retailers}

The combined direction associated with a transaction between a distributor and retailer pair, in turn, is given by expression (33) but now we guarantee that the evolution of the product transactions is proportional to (33) with the rate of proportionality (speed of adjustment) $\phi_{k h \bar{l}}^{j \hat{l}}>0, \quad \forall j, \hat{l}, k, h, \bar{l}$ and cannot yield negative values. Hence, letting $\dot{q}_{k h \bar{l}}^{j \hat{l}}$ denote the rate of change of the transaction between distributor $j \hat{l}$ and retailer $k h \bar{l}$, we obtain the following 
dynamics, which hold for all distributors and all retailers:

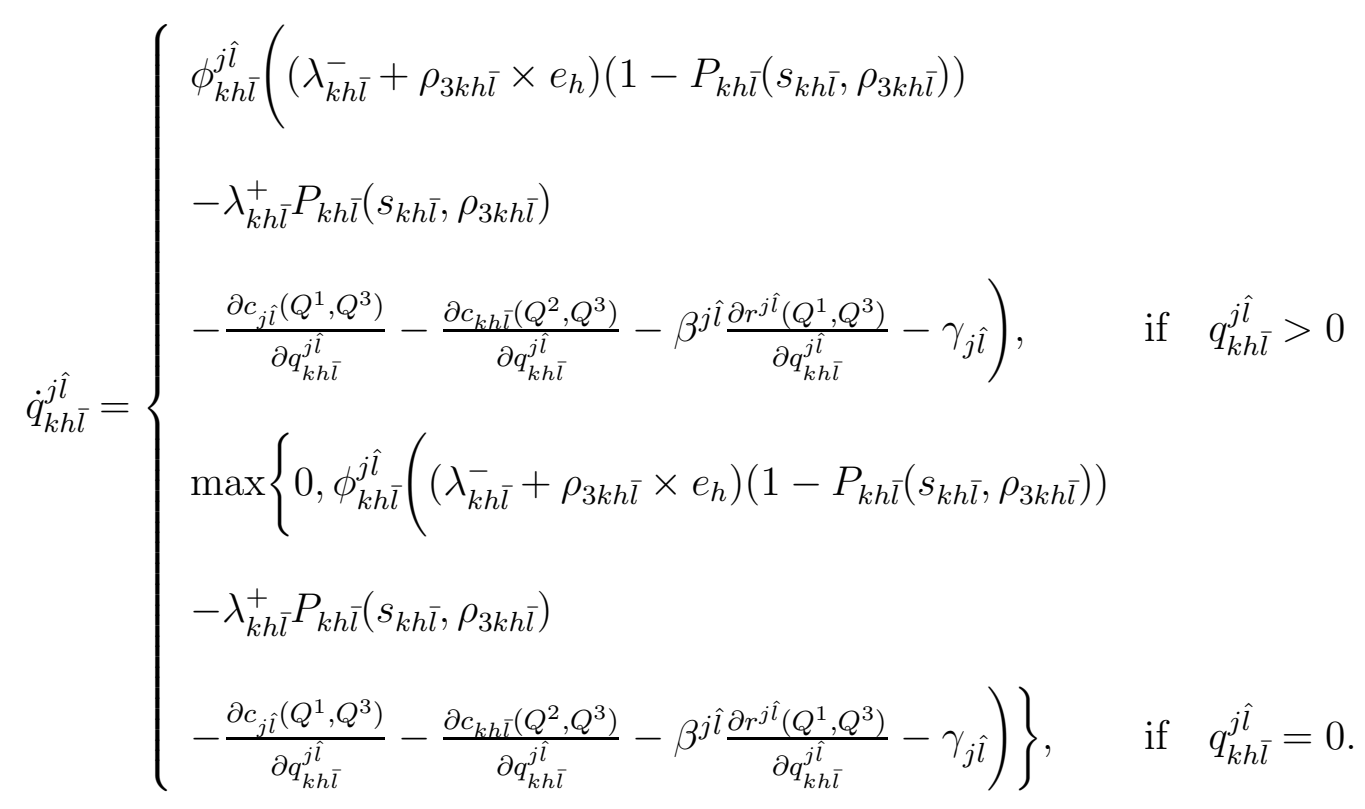

The volume of the product transacted between a distributor/retailer pair increases if the sum of the price that the consumers, in effect, are willing to pay at the retailer exceeds the various marginal costs, marginal risk associated with the distributor and the price charged at the distributor. If the latter sum exceeds the former price, then the volume of transaction will decrease.

\section{The Projected Dynamical System}

Consider now a dynamical system in which the retailer prices evolve according to (1) for all retailers $k h \bar{l}$; the prices at the distributors evolve according to (2) for all distributors $j \hat{l}$; the product transactions between the distributors and the retailers evolve according to (37), the product transactions between manufacturers and distributors evolve according to (35), and the product transactions between manufacturers and retailers evolve according to (36).

Let now $X$ denote the aggregate column vector $\left(Q^{1}, Q^{2}, Q^{3}, \gamma, \rho_{3}\right)$ in the feasible set $\mathcal{K} \equiv R_{+}^{I L J H L+I L K H L+J L K H L+J L+K H L}$. Define the column vector

$$
F(X) \equiv\left(F_{j h \hat{l}}^{i l}, F_{k h \bar{l}}^{i l}, F_{k h \bar{l}}^{j \hat{l}}, F_{j \hat{l}}, F_{k h \bar{l}}\right)_{i=1, \ldots, I ; l=\hat{l}=\bar{l}=1, \ldots, L ; j=1, \ldots, J ; h=1, \ldots, H ; k=1, \ldots, K},
$$

with components: $-F_{j h \hat{l}}^{i l}$ given by (21), $\forall i, l, j, h, \hat{l} ;-F_{k h \bar{l}}^{i l}$ given by $(35), \forall i, l, k, h, \bar{l} ;-F_{k h \bar{l}}^{j \hat{l}}$ given by (34), $\forall j, \hat{l}, k, h, \bar{l} ;-F_{j \hat{l}} \equiv \phi_{j \hat{l}}\left(\sum_{k=1}^{K} \sum_{h=1}^{H} \sum_{\bar{l}=1}^{L} q_{k h \hat{l}}^{j \hat{l}}-\sum_{i=1}^{I} \sum_{l=1}^{L} \sum_{h=1}^{H} q_{j h \hat{l}}^{i l}\right), \forall j, \hat{l}$; $-F_{k h \bar{l}} \equiv \phi_{k h \bar{l}}\left(d_{k h \bar{l}}\left(\rho_{3 k h \bar{l}}\right)-\sum_{i=1}^{I} \sum_{l=1}^{L} q_{k h \bar{l}}^{i l}-\sum_{j=1}^{J} \sum_{\hat{l}=1}^{L} q_{k h \bar{l}}^{j \hat{l}}\right), \forall k, h, \bar{l}$. 
Then the dynamic model described by (35), (36), (37), and (1), (2), for all $i, l, j, \hat{l}, k, h, \bar{l}$, can be rewritten as a projected dynamical system (PDS) (see Nagurney and Zhang (1996)) defined by the following initial value problem:

$$
\dot{X}=\Pi_{\mathcal{K}}(X,-F(X)), \quad X(0)=X_{0},
$$

where $\Pi_{\mathcal{K}}$ is the projection operator of $-F(X)$ onto $\mathcal{K}$ at $X$ and $X_{0}=\left(Q^{10}, Q^{20}, Q^{30}, \gamma^{0}, \rho_{3}^{0}\right)$ is the initial point corresponding to the initial product transaction and price pattern. Note that since the feasible set $\mathcal{K}$ is simply the nonnegative orthant the projection operation takes on a very simple form as revealed through (35), (36), (37), and (2), (1).

The trajectory of (38) describes the dynamic evolution of the product transactions between tiers of decision-makers in the supply chain supernetwork as well as the prices associated with the bottom two tiers of the network. The dynamical system (38) is non-classical since it has a discontinuous right-hand side due to the projection operation. Such dynamical systems were introduced by Dupuis and Nagurney (1993) and have been used to study a plethora of dynamic models in economics, finance, and transportation (see Nagurney and Zhang (1996)). In addition, the projected dynamical systems methodology has been used to-date to formulate dynamical supply chain network models, with and without electronic commerce (cf. Nagurney and Dong (2002) and the refereneces therein).

\section{A Stationary/Equilibrium Point}

We now discuss the stationary point of the projected dynamical system (38). Recall that a stationary point of a dynamical system is that point where $\dot{X}=0$ and, hence, in the context of our model, when there is no change in the product transactions in the supply chain supernetwork and no change in the prices.

The following result is immediate from the results in Dupuis and Nagurney (1993).

\section{Theorem 1: Set of Stationary Points Coincides with the Set of Solutions of a Variational Inequality Problem}

Since the feasible set $\mathcal{K}$ is a convex polyhedron, the set of stationary points of the projected dynamical system given by (38), that is, $X^{*}$ such that $0=\Pi_{\mathcal{K}}\left(X^{*},-F\left(X^{*}\right)\right)$, coincides with the set of solutions to the variational inequality problem given by: determine $X^{*} \in \mathcal{K}$, such that

$$
\left\langle F\left(X^{*}\right), X-X^{*}\right\rangle \geq 0, \quad \forall X \in \mathcal{K},
$$

where $F(X)$ and $X$ are as defined above and $\langle\cdot, \cdot\rangle$ denotes the inner product in $N$-dimensional 
Euclidian space where here $N=I L J H L+I L K H L+J L K H L+J L+K H L$, or, explicitly, we have that a product transaction and price pattern $X^{*} \equiv\left(Q^{1 *}, Q^{2 *}, Q^{3 *}, \gamma^{*}, \rho_{3}^{*}\right) \in \mathcal{K}$ is a stationary point of the projected dynamical system (38), if and only if it satisfies the variational inequality problem:

$$
\begin{aligned}
& \sum_{i=1}^{I} \sum_{l=1}^{L} \sum_{j=1}^{J} \sum_{h=1}^{H} \sum_{\hat{l}=1}^{L} \phi_{j h \hat{l}}^{i l}\left[\frac{\partial f^{i l}\left(Q^{1 *}, Q^{2 *}\right)}{\partial q_{j h \hat{l}}^{i l}}+\frac{\partial c_{j h \hat{l}}^{i l}\left(q_{j h \hat{l}}^{i l *}\right)}{\partial q_{j h \hat{l}}^{i l}}+\frac{\partial c_{j \hat{l}}\left(Q^{1 *}, Q^{3 *}\right)}{\partial q_{j h \hat{l}}^{i l}}+\alpha^{i l} \frac{\partial r^{i l}\left(Q^{1 *}, Q^{2 *}\right)}{\partial q_{j h \hat{l}}^{i l}}\right. \\
& \left.+\beta^{j \hat{l}} \frac{\partial r^{j \hat{l}}\left(Q^{1 *}, Q^{3 *}\right)}{\partial q_{j h \hat{l}}^{i l}}-\gamma_{j \hat{l}}^{*}\right] \times\left[q_{j h \hat{l}}^{i l}-q_{j h \hat{l}}^{i l}\right] \\
& +\sum_{i=1}^{I} \sum_{l=1}^{L} \sum_{k=1}^{K} \sum_{h=1}^{H} \sum_{\bar{l}=1}^{L} \phi_{k h \bar{l}}^{i l}\left[\frac{\partial f^{i l}\left(Q^{1 *}, Q^{2 *}\right)}{\partial q_{k h \bar{l}}^{i l}}+\frac{\partial c_{k h \bar{l}}^{i l}\left(q_{k k \bar{l}}^{i l *}\right)}{\partial q_{k h \bar{l}}^{i l}}+\frac{\partial c_{k h \bar{l}}\left(Q^{2 *}, Q^{3 *}\right)}{\partial q_{k h \bar{l}}^{i l}}+\alpha^{i l} \frac{\partial r^{i l}\left(Q^{1 *}, Q^{2 *}\right)}{\partial q_{k h \bar{l}}^{i l}}\right. \\
& \left.+\lambda_{k h \bar{l}}^{+} P_{k h \bar{l}}\left(s_{k h \bar{l}}^{*}, \rho_{3 k h \bar{l}}^{*}\right)-\left(\lambda_{k h \bar{l}}^{-}+\rho_{3 k h \bar{l}}^{*} \times e_{h}\right)\left(1-P_{k h \bar{l}}\left(s_{k h \bar{l}}^{*}, \rho_{3 k h \bar{l}}^{*}\right)\right)\right] \times\left[q_{k h \bar{l}}^{i l}-q_{k h \bar{l}}^{i l *}\right] \\
& +\sum_{j=1}^{J} \sum_{\hat{l}=1}^{L} \sum_{k=1}^{K} \sum_{h=1}^{H} \sum_{\bar{l}=1}^{L} \phi_{k h \bar{l}}^{j \hat{l}}\left[\lambda_{k h \bar{l}}^{+} P_{k h \bar{l}}\left(s_{k h \bar{l}}^{*}, \rho_{3 k h \bar{l}}^{*}\right)-\left(\lambda_{k h \bar{l}}^{-}+\rho_{3 k h \bar{l}}^{*} \times e_{h}\right)\left(1-P_{k h \bar{l}}\left(s_{k h \bar{l}}^{*}, \rho_{3 k h \bar{l}}^{*}\right)\right)\right. \\
& \left.+\frac{\partial c_{j \hat{l}}\left(Q^{1 *}, Q^{3 *}\right)}{\partial q_{k h \bar{l}}^{j \hat{l}}}+\frac{\partial c_{k h \bar{l}}\left(Q^{2 *}, Q^{3 *}\right)}{\partial q_{k h \bar{l}}^{j \hat{l}}}+\beta^{j \hat{l}} \frac{\partial r^{j \hat{l}}\left(Q^{1 *}, Q^{3 *}\right)}{\partial q_{k h \bar{l}}^{j \hat{l}}}+\gamma_{j \hat{l}}^{*}\right] \times\left[q_{k h \bar{l}}^{j \hat{l}}-q_{k h \bar{l}}^{j \hat{l}}\right] \\
& +\sum_{j=1}^{J} \sum_{\hat{l}=1}^{L} \phi_{j \hat{l}}\left[\sum_{i=1}^{I} \sum_{l=1}^{L} \sum_{h=1}^{H} q_{j h \hat{l}}^{i l *}-\sum_{k=1}^{K} \sum_{h=1}^{H} \sum_{\hat{l}=1}^{L} q_{k h \hat{l}}^{j \hat{l} *}\right] \times\left[\gamma_{j \hat{\imath}}-\gamma_{j \hat{l}}^{*}\right] \\
& +\sum_{k=1}^{K} \sum_{h=1}^{H} \sum_{\bar{l}=1}^{L} \phi_{k h \bar{l}}\left[\sum_{i=1}^{I} \sum_{l=1}^{L} q_{k h \bar{l}}^{i l *}+\sum_{j=1}^{J} \sum_{\hat{l}=1}^{L} q_{k h \bar{l}}^{j \hat{j} *}-d_{k h \bar{l}}\left(\rho_{3 k h \bar{l}}^{*}\right)\right] \times\left[\rho_{3 k h \bar{l}}-\rho_{3 k h \bar{l}}^{*}\right] \geq 0, \\
& \forall\left(Q^{1}, Q^{2}, Q^{3}, \gamma, \rho_{3}\right) \in \mathcal{K} .
\end{aligned}
$$

We now explore what can be said regarding the set of stationary points of the projected dynamical system (38), which coincides with the set of solutions to variational inequality (39), and the set of stationary points associated with a projected dynamical system in which all of the speeds of adjustment are equal to one (and the equivalent equilibria). First, we need to introduce some notation. We express the components of $F(X)$ above, for simplicity, as $F_{1}, \ldots, F_{N}$ and recall that the feasible set is the nonnegative orthant. We also denote the corresponding variational inequality by $\mathrm{VI}(F, \mathcal{K})$. 


\section{Theorem 2}

Assume that $\mathcal{K}$ is the convex polyhedron given by $R_{+}^{N}$ and that $\phi \equiv\left(\phi_{1}, \ldots, \phi_{N}\right)$ is a vector of positive terms. Then, the set of stationary points of the ordinary differential equation given in (38), which coincides with the set of solutions $X^{*}$ to variational inequality (39), are equivalent to the set of solutions to the variational inequality $\operatorname{VI}\left(F^{\prime}, \mathcal{K}\right)$ where:

$$
F^{\prime} \equiv\left(F_{1}^{\prime}, \ldots, F_{N}^{\prime}\right)
$$

and

$$
F \equiv\left(\phi_{1} F_{1}^{\prime}, \ldots, \phi_{N} F_{N}^{\prime}\right)
$$

Proof: From Theorem 1 we know that the set of stationary points of the ODE (38) coincides with the set of solutions to $\operatorname{VI}(F, \mathcal{K})$. We now prove that the solutions to $\operatorname{VI}(F, \mathcal{K})$ coincide with the solutions to $\mathrm{VI}\left(F^{\prime}, \mathcal{K}\right)$.

We first prove that if $X^{*}$ solves $\operatorname{VI}(F, \mathcal{K})$ then it also solves $\operatorname{VI}\left(F^{\prime}, \mathcal{K}\right)$. Since $X^{*} \equiv$ $\left(X_{1}^{*}, X_{2}^{*}, \ldots, X_{N}^{*}\right)$ is a solution to $\operatorname{VI}(F, \mathcal{K})$, then the following must hold, by definition:

$$
\begin{gathered}
\sum_{i=1}^{N} F_{i}\left(X^{*}\right) \times\left[X_{i}-X_{i}^{*}\right]=F_{1}\left(X^{*}\right) \times\left[X_{1}-X_{1}^{*}\right]+\ldots+F_{N}\left(X^{*}\right) \times\left[X_{N}-X_{N}^{*}\right] \\
=\phi_{1} F_{1}^{\prime}\left(X^{*}\right) \times\left[X_{1}-X_{1}^{*}\right]+\phi_{1} F_{2}^{\prime}\left(X^{*}\right) \times\left[X_{2}-X_{2}^{*}\right]+\ldots+\phi_{1} F_{N}^{\prime}\left(X^{*}\right) \times\left[X_{N}-X_{N}^{*}\right] \geq 0, \quad \forall X \in \mathcal{K} .
\end{gathered}
$$

Setting now $X_{2}=X_{2}^{*}, \ldots, X_{N}=X_{N}^{*}$ and substituting the resultants into (41), yields:

$$
\phi_{1} F_{1}^{\prime}\left(X^{*}\right) \times\left[X_{1}-X_{1}^{*}\right] \geq 0 \Leftrightarrow F_{1}^{\prime}\left(X^{*}\right) \times\left[X_{1}-X_{1}^{*}\right] \geq 0, \quad \forall X_{1} \in R_{+},
$$

and for any scalar $\phi_{1}>0$.

In a similar manner, we may set $X_{1}=X_{1}^{*}, X_{3}=X_{3}^{*}, \ldots, X_{N}=X_{N}^{*}$ with substitution into (41) yielding:

$$
\phi_{2} F_{2}^{\prime}\left(X^{*}\right) \times\left[X_{2}-X_{2}^{*}\right] \geq 0 \Leftrightarrow F_{2}^{\prime}\left(X^{*}\right) \times\left[X_{2}-X_{2}^{*}\right] \geq 0, \quad \forall X_{2} \in R_{+},
$$

and for any scalar $\phi_{2}>0$.

Proceeding in the same fashion with all the remaining $X_{i}$ variables, we obtain:

$$
F_{1}^{\prime}\left(X^{*}\right) \times\left[X_{1}-X_{1}^{*}\right] \geq 0, \quad \forall X_{1} \in R_{+}, \quad F_{2}^{\prime}\left(X^{*}\right) \times\left[X_{2}-X_{2}^{*}\right] \geq 0, \quad \forall X_{2} \in R_{+},
$$




$$
\ldots \quad F_{N}^{\prime}\left(X^{*}\right) \times\left[X_{N}-X_{N}^{*}\right] \geq 0, \quad \forall X_{N} \in R_{+} .
$$

Summation of all the inequalities in (44) yields:

$$
\sum_{i=1}^{N} F_{i}^{\prime}\left(X^{*}\right) \times\left[X_{i}-X_{i}^{*}\right]=\left\langle F^{\prime}\left(X^{*}\right), X-X^{*}\right\rangle \geq 0, \quad \forall X \in \mathcal{K} \equiv R_{+}^{N} .
$$

Hence, we have shown that $X^{*}$ is also a solution to $\operatorname{VI}\left(F^{\prime}, \mathcal{K}\right)$.

We now establish the converse, that is, if $X^{\prime}$ solves $\operatorname{VI}\left(F^{\prime}, \mathcal{K}\right)$, it also solves $\operatorname{VI}(F, \mathcal{K})$. Let $X^{\prime}$ be a solution to $\operatorname{VI}\left(F^{\prime}, \mathcal{K}\right)$. Then the following must hold:

$$
\sum_{i=1}^{N}\left[F_{i}^{\prime}\left(X^{\prime}\right)\right] \times\left[X_{i}-X_{i}^{\prime}\right]=F_{1}^{\prime}\left(X^{\prime}\right) \times\left[X_{1}-X_{1}^{\prime}\right]+\ldots+F_{N}^{\prime}\left(X^{\prime}\right) \times\left[X_{N}-X_{N}^{\prime}\right] \geq 0, \quad \forall X \in \mathcal{K}
$$

Setting now: $X_{2}=X_{2}^{\prime}, \ldots, X_{N}=X_{N}^{\prime}$ (all the variables except for the first) and substituting the resultants into (46), we obtain:

$$
F_{1}^{\prime}\left(X^{\prime}\right) \times\left[X_{1}-X_{1}^{\prime}\right] \geq 0 \Leftrightarrow \phi_{1} F_{1}^{\prime}\left(X^{\prime}\right) \times\left[X_{1}-X_{1}^{\prime}\right]=F_{1}\left(X^{\prime}\right) \times\left[X_{1}-X_{1}^{\prime}\right] \geq 0, \quad \forall X_{1} \in R_{+},
$$

and for any scalar $\phi_{1}>0$.

In a similar manner, we set: $X_{1}=X_{1}^{\prime}, X_{3}=X_{3}^{\prime}, \ldots, X_{N}=X_{N}^{\prime}$ with substitution into (46) yielding:

$$
F_{2}^{\prime}\left(X^{\prime}\right) \times\left[X_{2}-X_{2}^{\prime}\right] \geq 0 \Leftrightarrow \phi_{2} F_{2}^{\prime}\left(X^{\prime}\right) \times\left[X_{2}-X_{2}^{\prime}\right]=F_{2}\left(X^{\prime}\right) \times\left[X_{2}-X_{2}^{\prime}\right] \geq 0, \quad \forall X_{2} \in R_{+},
$$

and for any scalar $\phi_{2}>0$.

Proceeding in the same manner with all the remaining variables, we conclude that

$$
\begin{gathered}
F_{1}\left(X^{\prime}\right) \times\left[X_{1}-X_{1}^{\prime}\right] \geq 0, \quad \forall X_{1} \in R_{+}, \quad F_{2}\left(X^{\prime}\right) \times\left[X_{2}-X_{2}^{\prime}\right] \geq 0, \quad \forall X_{2} \in R_{+}, \\
\ldots \quad F_{N}\left(X^{\prime}\right) \times\left[X_{N}-X_{N}^{\prime}\right] \geq 0, \quad \forall X_{n} \in R_{+},
\end{gathered}
$$

which leads us to conclude that

$$
\sum_{i=1}^{N} F_{i}\left(X^{\prime}\right) \times\left[X_{i}-X_{i}^{\prime}\right]=\left\langle F\left(X^{\prime}\right), X-X^{\prime}\right\rangle \geq 0, \quad \forall X \in \mathcal{K} \equiv R_{+}^{N} .
$$

Therefore, $X^{\prime}$ solves $\operatorname{VI}(F, \mathcal{K})$.

Hence, we have established that the set of stationary points of:

$$
\dot{X}=\Pi_{\mathcal{K}}(X,-F(X)), \quad X(0)=X_{0} \in \mathcal{K},
$$


that is, $X^{*}$ satisfying:

$$
0=\Pi_{\mathcal{K}}\left(X^{*},-F\left(X^{*}\right)\right),
$$

coincides with the set of solutions of $\operatorname{VI}\left(F^{\prime}, \mathcal{K}\right)$.

This theoretical result can be summarized as follows. The set of equilibria of the global supply chain network are independent of the speeds of adjustment. Hence, if one is interested merely in the computation of the equilibrium product flow and price patterns one may compute the solution to either variational inequality problem (45) or to variational inequality problem (50). However, if one is interested in tracking the trajectories to the equilibrium state then one should include the speeds of adjustment. We also note that the factors which we term "speeds of adjustment" can also capture conversion factors, if need be.

To conclude this Section, we present several properties of the PDS defined by the initial value problem (38) that follow from the theory of projected dynamical systems.

\section{Theorem 3: Existence and Uniqueness}

Assume that function $F$ that enters the initial value problem (38) is Lipschitz continuous. Then, for any $X_{0} \in \mathcal{K}$, there exists a unique solution $X_{0}(t)$ to the initial value problem (38).

Proof: Follows from Theorem 2.5 in Nagurney and Zhang (1996).

\section{Definition 2: Stability of the System}

The system defined by (38) is stable if, for every $X_{0}$ and every equilibrium point $X^{*}$, the Euclidean distance $\left\|X^{*}-X_{0}(t)\right\|$ is a monotone nonincreasing function of time $t$.

Using Definition 2, a global stability result for the dynamical system (38) can be stated as follows:

\section{Theorem 4: Stability of the Global Supply Chain Network}

Assume that function $F$ that enters the initial value problem (38) is monotone. Then the dynamical system (38) underlying the global supply chain with risk and uncertainty is stable.

Proof: $F(X)$ is monotone and, hence, the conclusion follows directly from Theorem 4.1 of Zhang and Nagurney (1995). 


\section{The Euler Method}

In this Section, we consider the computation of the stationary points of (38). The algorithm that we propose is the Euler-type method, which is induced by the general iterative scheme of Dupuis and Nagurney (1993). It has been applied to-date to solve a plethora of dynamic network models (see, e.g., Nagurney and Zhang (1996) and Nagurney and Dong (2002)).

\section{The Euler Method}

\section{Step 0: Initialization}

Set $X^{0}=\left(Q^{10}, Q^{20}, Q^{30}, \gamma^{0}, \rho_{3}^{0}\right) \in \mathcal{K}$. Let $\mathcal{T}$ denote an iteration counter and set $\mathcal{T}=1$. Set the sequence $\left\{\alpha_{\mathcal{T}}\right\}$ so that $\sum_{\mathcal{T}=1}^{\infty} \alpha_{\mathcal{T}}=\infty, \alpha_{\mathcal{T}}>0, \alpha_{\mathcal{T}} \rightarrow 0$, as $\mathcal{T} \rightarrow \infty$ (which is a requirement for convergence).

\section{Step 1: Computation}

Compute $X^{\mathcal{T}}=\left(Q^{1 \mathcal{T}}, Q^{2 \mathcal{T}}, Q^{3 \mathcal{T}}, \gamma^{\mathcal{T}}, \rho_{3}^{\mathcal{T}}\right) \in \mathcal{K}$ by solving the variational inequality subproblem:

$$
\left\langle X^{\mathcal{T}}+\alpha_{\mathcal{T}} F\left(X^{\mathcal{T}-1}\right)-X^{\mathcal{T}-1}, X-X^{\mathcal{T}}\right\rangle \geq 0, \quad \forall X \in \mathcal{K} .
$$

\section{Step 2: Convergence Verification}

If $\left|X^{\mathcal{T}}-X^{\mathcal{T}-1}\right| \leq \epsilon$, with $\epsilon>0$, a pre-specified tolerance, then stop; otherwise, set $\mathcal{T}:=\mathcal{T}+1$, and go to Step 1.

Convergence results for the Euler method can be found in Dupuis and Nagurney (1993) and in the book by Nagurney and Zhang (1996).

Due to the fact that the feasible set underlying the global supply chain network model is that of the nonnegative orthant, the solution of (40) for our model takes on a very simple form for computational purposes.

\section{Computation of Product Transactions between Tiers Using Explicit Formulae}

In particular, we have that the production transactions between tiers of decision-makers in the global supply chain supernetwork can be computed explicitly and in closed form as 
follows for a given iteration $\mathcal{T}$ :

$$
\begin{gathered}
q_{j h \hat{l}}^{i l \mathcal{T}}=\max \left\{0, q_{j h \hat{l}}^{i l \mathcal{T}-1}-\alpha^{\mathcal{I}} \phi_{j h \hat{l}}^{i l}\left(\frac{\partial f^{i l}\left(Q^{1 \mathcal{T}-1}, Q^{2 \mathcal{T}-1}\right)}{\partial q_{j h \hat{l}}^{i l}}+\frac{\partial c_{j h \hat{l}}^{i l}\left(q_{j h \hat{l}}^{i l \mathcal{T}-1}\right)}{\partial q_{j h \hat{l}}^{i l}}+\frac{\partial c_{j \hat{l}}\left(Q^{1 \mathcal{T}-1}, Q^{3 \mathcal{T}-1}\right)}{\partial q_{j h \hat{l}}^{i l}}\right.\right. \\
\left.\left.+\alpha^{i l} \frac{\partial r^{i l}\left(Q^{1 \mathcal{T}-1}, Q^{2 \mathcal{T}-1}\right)}{\partial q_{j h \hat{l}}^{i l}}+\beta^{j \hat{l}} \frac{\partial r^{j \hat{l}}\left(Q^{1 \mathcal{T}-1}, Q^{3 \mathcal{T}-1}\right)}{\partial q_{j h \hat{l}}^{i l}}-\gamma_{j \hat{l}}^{\mathcal{T}-1}\right)\right\}, \quad \forall i, l, j, h, \hat{l} ; \\
q_{k h \bar{l}}^{i l \mathcal{T}}=\max \left\{0, q_{k h \bar{l}}^{i l \mathcal{T}-1}-\alpha^{\mathcal{T}} \phi_{k h \bar{l}}^{i l}\left(\frac{\partial f^{i l}\left(Q^{1 \mathcal{T}-1}, Q^{2 \mathcal{T}-1}\right)}{\partial q_{k h \bar{l}}^{i l}}+\frac{\partial c_{k h \bar{l}}^{i l}\left(q_{k h \bar{l}}^{i l \mathcal{T}-1}\right)}{\partial q_{k h \bar{l}}^{i l}}+\frac{\partial c_{k h \bar{l}}\left(Q^{2 \mathcal{T}-1}, Q^{3 \mathcal{T}-1}\right)}{\partial q_{k h \bar{l}}^{i l}}\right.\right. \\
\left.\left.+\alpha^{i l} \frac{\partial r^{i l}\left(Q^{1 \mathcal{T}-1}, Q^{2 \mathcal{T}-1}\right)}{\partial q_{k h \bar{l}}^{i l}}+\lambda_{k h \bar{l}}^{+} P_{k h \bar{l}}\left(s_{k h \bar{l}}^{\mathcal{T}-1}, \rho_{3 k h \bar{l}}^{\mathcal{T}-1}\right)-\left(\lambda_{k h \bar{l}}^{-}+\rho_{3 k h \bar{l}}^{\mathcal{T}-1} \times e_{h}^{\mathcal{T}-1}\right)\left(1-P_{k h \bar{l}}\left(s_{k h \bar{l}}^{\mathcal{T}-1}, \rho_{3 k h \bar{l}}^{\mathcal{T}-1}\right)\right)\right)\right\}, \\
\forall i, l, k, h, \bar{l} ;
\end{gathered}
$$

and

$$
\begin{gathered}
q_{k h \bar{l}}^{j \hat{l} \mathcal{T}}=\max \left\{0, q_{k h \bar{l}}^{j \hat{\mathcal{T}}-1}-\alpha^{\mathcal{T}} \phi_{k h \bar{l}}^{j \hat{l}}\left(\lambda_{k h \bar{l}}^{+} P_{k h \bar{l}}\left(s_{k h \bar{l}}^{\mathcal{T}-1}, \rho_{3 k h \bar{l}}^{\mathcal{T}-1}\right)-\left(\lambda_{k h \bar{l}}^{-}+\rho_{3 k h \bar{l}}^{\mathcal{T}-1} \times e_{h}^{\mathcal{T}-1}\right)\left(1-P_{k h \bar{l}}\left(s_{k h \bar{l}}^{\mathcal{T}-1}, \rho_{3 k h \bar{l}}^{\mathcal{T}-1}\right)\right)\right.\right. \\
\left.\left.+\frac{\partial c_{j \hat{l}}\left(Q^{1 \mathcal{T}-1}, Q^{3 \mathcal{T}-1}\right)}{\partial q_{k h \bar{l}}^{j \hat{l}}}+\frac{\partial c_{k h \bar{l}}\left(Q^{2 \mathcal{T}-1}, Q^{3 \mathcal{T}-1}\right)}{\partial q_{k h \bar{l}}^{j \hat{l}}}+\beta^{j \hat{l}} \frac{\partial \partial^{j \hat{l}}\left(Q^{1 \mathcal{T}-1}, Q^{3 \mathcal{T}-1}\right)}{\partial q_{k h \bar{l}}^{j \hat{l}}}+\gamma_{j \hat{l}}^{\mathcal{T}-1}\right)\right\}, \\
\forall \forall, \hat{l}, k, h, \bar{l} .
\end{gathered}
$$

\section{Computation of Product Prices Using Explicit Formulae}

Similarly, the prices associated with the middle-tier and bottom tier of nodes of the global supply chain supernetwork can also be computed explicitly and in closed form, at a given iteration $\mathcal{T}$ according to:

$$
\gamma_{j \hat{l}}^{\mathcal{T}}=\max \left\{0, \gamma_{j \hat{l}}^{\mathcal{T}-1}-\alpha^{\mathcal{T}} \phi_{j \hat{l}}\left(\sum_{h=1}^{H} \sum_{i=1}^{I} \sum_{l=1}^{L} q_{j h \hat{l}}^{i l \mathcal{T}-1}-\sum_{h=1}^{H} \sum_{k=1}^{K} \sum_{\hat{l}=1}^{L} q_{k h \hat{l}}^{j \hat{l} \mathcal{T}-1}\right)\right\}, \quad \forall j, \hat{l},
$$

and

$$
\rho_{3 k h \bar{l}}^{\mathcal{T}}=\max \left\{0, \rho_{3 k h \bar{l}}^{\mathcal{T}-1}-\alpha^{\mathcal{T}} \phi_{k h \bar{l}}\left(\sum_{i=1}^{I} \sum_{l=1}^{L} q_{k h \bar{l}}^{i l \mathcal{T}-1}+\sum_{j=1}^{J} \sum_{\hat{l}=1}^{L} q_{k h \bar{l}}^{j \hat{l} \mathcal{T}-1}-d_{k h \bar{l}}\left(\rho_{3 k h \bar{l}}^{\mathcal{T}-1}\right)\right)\right\}, \quad \forall k, h, \bar{l} .
$$

Moreover, the above computations can be done simultaneously and independently. The Euler method, hence, provides us with a discrete-time adjustment process by which the network decision-makers adjust their product transactions and the prices associated with the middle and bottom tiers of the network also adjust. 


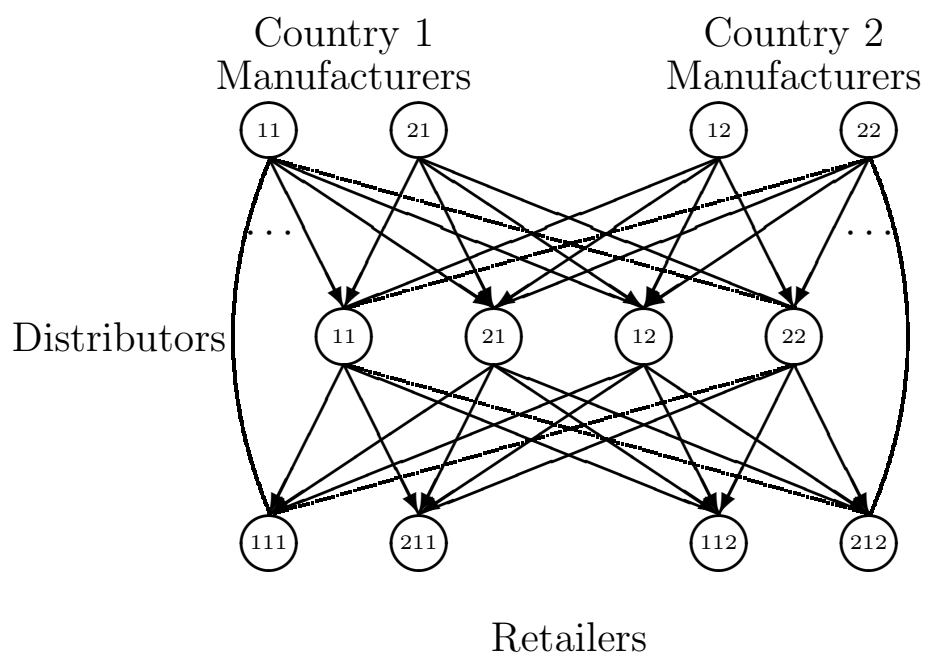

Figure 2: Global supply chain supernetwork for the numerical examples

\section{Numerical Examples}

In this Section, we apply the Euler method described in Section 3 to several numerical examples. The Euler method was coded in FORTRAN and the computer used was a SUN system at the University of Massachusetts at Amherst. The convergence criterion utilized was that the absolute value of the product transactions and prices between two successive iterations differed by no more than $10^{-5}$. The parameter $\alpha_{\mathcal{T}}$ in the Euler method (cf. (52)) was set to $.1\left\{1, \frac{1}{2}, \frac{1}{2}, \frac{1}{3}, \frac{1}{3}, \frac{1}{3}, \ldots\right\}$ for all the examples.

The structure of the global supply chain network for the examples is given in Figure 2. Specifically, we assumed that there were two countries, with two manufacturers in each country, and two distributors in each country. In addition, we assumed a single currency (for example, the euro) and two retailers in each country. Note that electronic transactions were permitted between the manufacturers and the retailers. Hence, we had that $I=2, L=2$, $J=2, K=2$, and $H=1$.

In all the examples, we assumed that the demands associated with the retail outlets followed a uniform distribution. In particular, we assumed that the random demand $\hat{d}_{k h \bar{l}}\left(\rho_{3 k h \bar{l}}\right)$, 
of retailer $k h \bar{l}$, is uniformly distributed in $\left[0, \frac{b_{k h \bar{l}}}{\rho_{3 k h \bar{l}}}\right]$, with $b_{k h \bar{l}}>0 ; k=1,2 ; h=1$, and $\bar{l}=1,2$. Therefore, we have that

$$
\begin{gathered}
P_{k h \bar{l}}\left(x, \rho_{3 k h \bar{l}}\right)=\frac{x \rho_{3 k h \bar{l}}}{b_{k h \bar{l}}} ; \quad k=1,2 ; h=1 ; \bar{l}=1,2, \\
F_{k h \bar{l}}\left(x, \rho_{3 k h \bar{l}}\right)=\frac{\rho_{3 k h \bar{l}}}{b_{k h \bar{l}}} ; \quad k=1,2 ; h=1 ; \bar{l}=1,2 ; \\
d_{k h \bar{l}}\left(\rho_{3 k h \bar{l}}\right)=E\left(\hat{d}_{k h \bar{l}}\right)=\frac{1}{2} \frac{b_{k h \bar{l}}}{\rho_{3 k h \bar{l}}} ; \quad k=1,2 ; h=1 ; \bar{l}=1,2 .
\end{gathered}
$$

It is straightforward to verify that the expected demand function $d_{k h \bar{l}}\left(\rho_{3 k h \bar{l}}\right)$ associated with retailer $k h \bar{l}$ is a decreasing function of the price at the demand market in the particular country. Since there was only one currency in each of the examples, we set $e_{h}=e_{1}=1$.

The Euler method was initialized as follows: all variables were set equal to zero, except for the initial retail prices $\rho_{3 k h \bar{l}}$, which were set to 1 for all $k, h, \bar{l}$.

\section{Example 1 and Variants}

The data for this example were constructed for easy interpretation purposes. The production cost functions of the manufacturers in the two countries (cf. (3)) were given, respectively, by:

$$
\begin{array}{ll}
f^{11}(q)=2.5\left(q^{11}\right)^{2}+q^{11} q^{21}+2 q^{11}, & f^{21}(q)=2.5\left(q^{21}\right)^{2}+q^{11} q^{21}+2 q^{21} \\
f^{12}(q)=2.5\left(q^{12}\right)^{2}+q^{12} q^{22}+2 q^{12}, & f^{22}(q)=2.5\left(q^{12}\right)^{2}+q^{12} q^{22}+2 q^{22}
\end{array}
$$

The transaction costs faced by the manufacturers and associated with transacting with the distributors (cf. (4a)) were given by:

$$
c_{j h \hat{l}}^{i l}=.5\left(q_{j h \hat{l}}^{i l}\right)^{2}+3.5 q_{j h \hat{l}}^{i l}, \quad \text { for } i=1,2 ; l=1,2 ; j=1,2 ; h=1 ; \hat{l}=1,2 .
$$

The transaction costs faced by the manufacturers but associated with transacting with the retailers electronically (cf. (4b)) were given by:

$$
c_{k h \bar{l}}^{i l}=.5\left(q_{k h \bar{l}}^{i l}\right)^{2}+5 q_{k h \bar{l}}^{i l}, \quad \text { for } i=1,2 ; l=1,2 ; k=1,2 ; h=1 ; \bar{l}=1,2 .
$$

The handling costs of the distributors in the two countries, in turn, (cf.(12b), were given by:

$$
c_{j \hat{l}}=.5\left(\sum_{i=1}^{2} \sum_{l=1}^{2} q_{j h \hat{l}}^{i l}\right)^{2}, \quad \text { for } j=1,2 ; \hat{l}=1,2 .
$$


The handling costs of the retailers (cf. (22)) were:

$$
c_{k h \bar{l}}=.5\left(\sum_{j=1}^{2} \sum_{\hat{l}=1}^{2} q_{k h \bar{l}}^{j \hat{l}}\right)^{2}, \quad \text { for } k=1,2 ; h=1 ; \bar{l}=1,2 .
$$

The $b_{k h l} \mathrm{~s}$ (cf. (58) - (60)) were set to 100 for all $k, h, \bar{l}$. The weights associated with excess supply and with excess demand at the retailers were (see following (26)): $\lambda_{k h \bar{l}}^{+}=\lambda_{k h \bar{l}}^{-}=1$ for $k=1,2 ; h=1$, and $\bar{l}=1,2$. Thus, we assigned equal weights for each retailer in each country for excess supply and excess demand.

In Example 1, we set all the weights associated with risk minimization to zero, that is, we had that $\alpha^{i l}=0$ for $i=1,2$ and $l=1,2$ and $\beta^{j \hat{l}}=0$ for $j=1,2$ and $\hat{l}=1,2$. This means that in the first example all the manufacturers and all the distributors were concerned with profit maximization exclusively.

We also assumed that all the speed adjustment parameters were identically equal to 1 .

The Euler converged in 125, 252 iterations and yielded the following equilibrium pattern. All physical transactions were equal to .186 , that is, we had that $q_{j h \hat{l}}^{i l *}=q_{k h \grave{l}}^{j \hat{l} *}=.186$ for all $i, l$, $j, h, \hat{l}$, and $k, \bar{l}$. All product transactions conducted electronically via the Internet, in turn, were equal to .175 , that is, we had that $q_{k h \bar{l}}^{i l *}=.175$ for all $i, l, k, h, \bar{l}$. Note that there was a larger volume of product transacted physically than electronically in this example.

The computed equilibrium prices, in turn, were as follows. The equilibrium prices at the distributors were: $\gamma_{j \hat{l}}^{*}=15.097$ for $j=1,2$ and $\hat{l}=1,2$, whereas the demand market equilibrium prices were: $\rho_{3 k h \bar{l}}^{*}=32.88$ for $k=1,2, h=1$, and $\bar{l}=1,2$. Note that, as expected, the demand market prices exceed the prices for the product at the distributor level. This is due to the fact that the prices increase as the product propagates down through the supply chain since costs accumulate.

We now made the following change to the data in order to explore the effects of different speeds of adjustment: we increased the speeds of adjustment associated with the demand market prices so that the $\phi_{k h l}$ s were now all equal to 10. The Euler method converged to essentially the identical equilibrium product flow and price pattern except that now the Euler method only required 34, 270 iterations.

We then conducted another numerical exercise. We returned to the original data for Example but now we assumed that all the speeds of adjustment increased to 5. Again, as predicted by Theorem 2, the equilibrium pattern does not change. However, the Euler method now required only 27,265 iterations for convergence. 


\section{Example 2 and Variants}

Example 2 was constructed from Example 1 as follows. All the data were as in the baseline Example 1 except that now we set the $b_{k h l} \mathrm{~s}=1000$. This means (cf. (58) - (60)) that, in effect, the demand has increased for the product at all retailers in all countries.

The Euler method converged, again in 125, 252 iterations, and yielded the following new equilibrium pattern: the product transactions between the manufacturers and the distrib-

utors were: $q_{j h \hat{l}}^{i l *}=.286$ for all $i, l, j, h, \hat{l}$; whereas the volumes of the product transacted electronically between the manufacturers and the retailers were: $q_{k h \bar{l}}^{i l *}=1.07$ for all $i, l$ and $k, h, \bar{l}$. Hence, the volumes of electronic transactions exceeded the physical ones. Finally, the computed equilibrium product transactions between the distributors and the retailers were: $q_{k h \bar{l}}^{j \hat{l} *}=.286$ for all $j, \hat{l}$ and $k, h, \bar{l}$.

The computed equilibrium prices associated with the distributors, in turn, were: $\gamma_{j \hat{l}}^{*}=$ 39.46 for $j=1,2$ and $\hat{l}=1,2$, whereas the equilibrium demand market prices were: $\rho_{3 k h \bar{l}}^{*}=$ 90.31 for $k=1,2 ; h=1$, and $\bar{l}=1,2$.

Note that since the demand increased, the product transactions also increased. In this example, there were more transactions conducted electronically than physically. Also, observe that since demand increased, the demand prices also increased as did the prices at the distributors.

We then modified Example 2 as follows. We increased the speeds of adjustment associated with the demand market prices so that the $\phi_{k h l} \mathrm{~s}$ were now all equal to 10. Again, as predicted by the theory, the equilibrium pattern was essentially the same. However, the number of iterations required for convergence decreased to 40, 258.

Finally, we constructed another example using Example 2 as a baseline as follows. We now increased all the speeds of adjustment to 5 . The number of iterations now decreased to 17, 030. Note that these results do follow intuition in that one would expect the speeds of adjustment to affect the behavior of the system, and, therefore, the number of iterations.

\section{Example 3 and Variants}

Example 3 was constructed from baseline Example 2 as follows. We kept the data as in Example 2 but we assumed now that the first manufacturer in the first country was a multicriteria decision-maker and concerned with risk minimization with his risk function 
being given by:

$$
r^{11}=\left(\sum_{k h \bar{l}} q_{k h \bar{l}}^{11}-2\right)^{2},
$$

that is, the manufacturer sought to achieve, in a sense, a certain goal target associated with his electronic transactions. The weight associated with his risk measure was $\alpha^{11}=1$.

The Euler method yielded the following new equilibrium pattern (and again in 125, 252 iterations). The computed equilibrium product transactions between the first manufacturer in the first country and the distributors were now: $q_{j h \hat{l}}^{11 *}=.700$, for $j=1,2, h=1$, and $\hat{l}=$ 1,2 . The analogous transactions, but from the second manufacturer in the first country were; $q_{j h l}^{21 *}=.185$. All other product transactions between the manufacturers and the distributors were equal to .182 .

The equilibrium product transactions associated with electronic transactions were as follows. For the first manufacturer in the first country (who is now concerned with risk associated with electronic transactions) the product transactions were: $q_{k h \bar{l}}^{11 *}=.632$, for $k=1,2$, $h=1, \bar{l}=1,2$. The analogous transactions but from the second manufacturer in the first country, were all 1.185, with the remainder of the electronic transactions at equilibrium also equal to 1.182. Hence, the first manufacturer in the first country reduced the volume of his transactions conducted electronically since there was increased risk associated with such transactions.

The product transactions at equilibrium between the distributors and the retailers, in turn, were: $q_{k h \bar{l}}^{j \hat{l} *}=.314$, for $j=1,2, k=1,2, h=1$, and $\bar{l}=1,2$.

The equilibrium prices were now: $\gamma_{j \hat{l}}^{*}=39.50$ for $j=1,2 ; \hat{l}=1,2$ whereas $\rho_{3 k h \bar{l}}^{*}=90.53$ for $k=1,2 ; h=1$, and $\bar{l}=1,2$.

We then proceeded to construct the following variant of Example 3: we doubled all the adjustment parameters associated with the product transactions. Interestingly, the number of iterations did not change and this may be that the global supply chain network model is more sensitive to the consumers associated with the demand markets and the prices that they are will to pay for the product (which seems quite reasonable).

Finally, we doubled all the speeds of adjustment and the number of iterations now decreased to 59,628 .

Note that, interestingly, the number of iterations required for convergence of the Euler method was identical for the baseline Examples 1, 2, and 3. When we relaxed the convergence tolerance from $10^{-5}$ to $10^{-4}$ the corresponding number of iterations for these three 
examples were now reduced from 125, 252 iterations to, respectively: 33, 672; 51,901, and 52, 274 iterations. Although the computed product flows did not change much, the computed demand market prices were, under the more relaxed convergence criterion, respectively, for Example 1, Example 2, and Example 3: 29.404; 86.822, and 87.036 (for all the demand markets in each case). Hence, the algorithm is sensitive to the form of the demand functions in the numerical examples above. Nevertheless, convergence was obtained.

Clearly, the above examples are stylized but they, nevertheless, demonstrate the efficacy of the model and the computational procedure. One may now conduct numerous simulations by modifying the data as well as adding decision-makers with their associated functions and weights and investigating the effects on the equilibrium product transactions and product prices.

Moreover, it would be interesting to conduct additional computational experiments.

\section{Summary and Conclusions}

In this paper, we have developed a dynamic, multitiered global supply chain supernetwork model consisting of manufacturers, distributors, and retailers and analyzed it both from theoretical and computational perspectives. The model allows for electronic commerce and handles decision-making under risk and uncertainty. Specifically, the manufacturers as well as the distributors are assumed to be multicriteria decision-makers and concerned not only with profit maximization but also with risk minimization. The demands for the product, in turn, are random. In addition, the model includes distinct speeds of adjustment.

The framework permits for the handling of as many countries, as many manufacturers in each country, as many currencies in which the product can be obtained, and as many retailers, as required by the specific application. Moreover, the generality of the framework allows for the demand to have almost any distribution as long as it satisfies certain technical conditions. In addition, the retailers need not be country-specific and can transact either virtually or physically with both the manufacturers and the consumers.

The dynamic model, which is formulated as a projected dynamical system, provides the evolution of the product transactions between tiers of the global supply chain network as well as the prices associated with the product at the retailer and at the distributor levels. The model substantially generalizes the recent work of Nagurney, Cruz, and Matsypura (2003) to include supply-side risk and demand-side risk.Finally, the new theoretical results show that the speeds of adjustment do not alter the set of stationary points of the dynamical model 
nor the equilibria of the corresponding variational inequality formulation.

The results in this paper add to the timely topic of rigorous model and quantitative tool development for combined global supply chain network analysis and risk management. 


\section{References}

Arrow, K. J. and L. Hurwicz (1958), "On the Stability of the Competitive Equilibrium, I," Econometrica 26(4), 522-552.

Chankong, V. and Y. Y. Haimes (1997), Multiobjective Decision Making: Theory and Methodology, North-Holland, New York.

Cohen, M. A. and A. Huchzermeier (1998), "Global Supply Chain Management: A Survey of Research and Applications," in Quantitative Models for Supply Chain Management, S. Tayur, M. Magazine, and R. Ganeshan, editors, Kluwer Academic Publishers, New York, pp. $669-702$.

Cohen, M. A. and S. Mallik (1997), "Global Supply Chains: Research and Applications," Production and Operations Management 6, 193-210.

Dong, J., D. Zhang and A. Nagurney (2004), "A Supply Chain Network Equilibrium Model with Random Demands," European Journal of Operational Research 156, 194-212.

Dupuis, P. and A. Nagurney (1993), "Dynamical Systems and Variational Inequalities," Annals of Operations Research 44, 9-42.

Engardio, P., M. Shari, A. Weintraub and C. Arnst (2003), Deadly Virus, BusinessWeek Online, April 14.

Fishburn, P. C. (1970), Utility Theory for Decision Making, John Wiley \& Sons, New York.

Fisher, F. M. (1961), "The Stability of the Cournot Oligopoly Solution: The Effects of Speeds of Adjustment and Increasing Marginal Costs," The Review of Economic Studies 28(2), 125-135.

Hahn, F. H. (1970), "Some Adjustment Problems," Econometrica 38(1), 1-17.

Hicks, J. R. (1939), Value and Capital, London, England.

Huchzermeier, A. and M. A. Cohen (1996), "Valuing Operational Flexibility Under Exchange Rate Uncertainty," Operations Research 44, 100-113.

Keeney, R. L. and H. Raiffa (1993), Decisions with Multiple Objectives: Preferences and Value Tradeoffs, Cambridge University Press, Cambridge, England. 
Kogut, B. and N. Kulatilaka (1994), "Options Thinking and Platform Investments: Investing in Opportunity," California Management Review 36, No. 4, 52-71.

Metzler, L. A. (1945), "Stability of Multiple Markets: The Hicks Conditions," Econometrica 13(4), 277-292.

Nagurney, A. (1999), Network Economics: A Variational Inequality Approach, second and revised edition, Kluwer Academic Publishers, Dordrecht, The Netherlands.

Nagurney, A. and J. Cruz (2003), "International Financial Networks with Electronic Transactions," in Innovations in Financial and Economic Networks, A. Nagurney, editor, Edward Elgar Publishers, Cheltenham, England, pp. 195-211.

Nagurney, A., J. Cruz and D. Matsypura (2003), "Dynamics of Global Supply Chain Supernetworks," Mathematical and Computer Modelling 37, 963-983.

Nagurney, A. and J. Dong (2002), Supernetworks: Decision-Making for the Information Age, Edward Elgar Publishers, Cheltenham, England.

Nagurney, A., J. Dong and D. Zhang (2002), "A Supply Chain Network Equilibrium Model," Transportation Research E 38, 281-303.

Nagurney, A. and K. Ke (2003), "Financial Networks with Electronic Transactions: Modeling, Analysis, and Computations," Quantitative Finance 3, 71-87.

Nagurney, A. and D. Zhang (1996), Projected Dynamical Systems and Variational Inequalities with Applications, Kluwer Academic Publishers, Boston, Massachusetts.

Nash, J. F. (1950), "Equilibrium Points in N-Person Games," in: Proceedings of the National Academy of Sciences, USA 36, 48-49.

Nash, J. F. (1951), "Noncooperative Games," Annals of Mathematics 54, 286-298.

Okuguchi, K. (1976), Expectations and Stability in Oligopoly models, SpringerVerlag, Berlin; New York.

Quandt, R. E. (1967), "On the Stability of Price Adjusting Oligopoly," Southern Economic Journal 33(3), 332-336.

Reinhardt, A. and N. Majidi (2003), "Europe's Borderless Market: The Net," BusinessWeek Online, May 12. 
Salkever, A. (2003), "Up and Comer: Intel's Sandra Morris," BusinessWeek Online, May 29.

Samuelson, P. A. (1941), "The Stability of Equilibrium: Comparative Statics and Dynamics," Econometrica 9(2), 97-120.

Sheffi, Y. (2001), "Supply Chain Management under the Threat of International Terrorism," The International Journal of Logistics Management 12, 1-11.

Yu, P. L. (1985), Multiple Criteria Decision Making - Concepts, Techniques, and Extensions, Plenum Press, New York.

Zhang, D. and A. Nagurney (1995), "On the Stability of Projected Dynamical Systems," Journal of Optimization Theory and Applications 85, 97-124.

Zhang, D. and A. Nagurney (1996), "Stability Analysis of an Adjustment Process for Oligopolistic Market Equilibrium Modeled as a Projected Dynamical System," Optimization 36, 263285. 